\title{
A comparison of the quick Sequential [Sepsis-related] Organ Failure Assessment score and the National Early Warning Score in non-ICU patients with/without infection
}

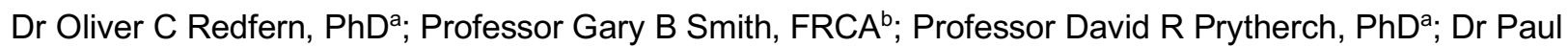
Meredith, PhDc; Dr Matthew Inada-Kim, FRCPd; Dr Paul E Schmidt, MRCPe

${ }^{a}$ Centre for Healthcare Modelling \& Informatics, University of Portsmouth, Portsmouth, UK

${ }^{b}$ Faculty of Health and Social Sciences, University of Bournemouth, Bournemouth, UK

${ }^{c}$ Research \& Innovation Department, Portsmouth Hospitals NHS Trust, Portsmouth, UK

${ }^{\mathrm{d}}$ Acute Medical Unit, Hampshire Hospitals NHS Foundation Trust, Winchester, UK

e Acute Medical Unit, Portsmouth Hospitals NHS Trust, Portsmouth, UK

\section{Work performed at Portsmouth Hospitals NHS Trust \& University of Portsmouth} No reprints will be available

\section{Corresponding author:}

Professor G B Smith, FRCA, FRCP,

Centre of Postgraduate Medical Research \& Education (CoPMRE),

Faculty of Health and Social Sciences,

Bournemouth University, Royal London House,

Christchurch Road, Bournemouth,

Dorset BH1 3LT, United Kingdom

Tel: +44 (0) 1202 962782; Fax: +44 (0) 1202962218

Email: gbsresearch@virginmedia.com

Conflicts of Interest and Source of Funding: VitalPAC, the system used to collect vital signs data in the submitted research project, is a collaborative development of The Learning Clinic Ltd (TLC) and Portsmouth Hospitals NHS Trust (PHT). At the time of the research, PHT had a royalty agreement with TLC to pay for the use of PHT intellectual property within the VitalPAC product. Dr Schmidt, and Dr Meredith are employed by PHT. Professor Smith was an employee of PHT until 31/03/2011. Professor Prytherch was an employee of PHT until 31/07/2016. Until October 2015, Dr. Schmidt and the wives of Professors Smith and Prytherch were minority shareholders in TLC. Until October 2015, Dr. Schmidt, Professor Smith and Professor Prytherch were unpaid research advisors to TLC and received reimbursement of travel expenses from TLC for attending symposia in the UK. Professor Smith is a member of the Royal College of Physicians of London's National Early Warning Score (NEWS) Development and Implementation Group (NEWSDIG), which developed NEWS. Professor Prytherch assisted the Royal College of Physicians of London in the analysis of data validating NEWS. Dr InadaKim is the UK National Clinical Advisor for Sepsis and one of the authors of the publication describing the use of routine data to define and measure suspicion of sepsis (SOS) that is used in the current research. Dr Inada-Kim is also UK National Clinical Advisor to the NHS for deterioration. Dr Redfern declares no conflicts of interest.

Funding: There was no funding for this study.

Word count $=3009$

Abstract word count $=235$

Number of references $=42$

Figures $=1$

Tables $=4$

Supplementary Figures $=6 ;$ Supplementary Tables $=6$

Key words: infection; sepsis; quick Sepsis-related Organ Failure Assessment; early warning scores; National Early Warning Score. 


\section{ABSTRACT}

Objective: The Sepsis-3 task force recommended the quick Sequential [Sepsis-Related] Organ Failure Assessment score (qSOFA) for identifying patients with suspected infection who are at greater risk of poor outcomes, but many hospitals already use the National Early Warning Score (NEWS) to identify high-risk patients, irrespective of diagnosis. We sought to compare the performance of qSOFA and NEWS in hospitalized, non-ICU patients with and without an infection.

Design: Retrospective cohort study.

Setting: Large UK General Hospital.

Patients: Adults hospitalised between $1^{\text {st }}$ January 2010 and $1^{\text {st }}$ February 2016.

Interventions: None

Measurements and Main Results: We applied the qSOFA score and NEWS to 5,435,344 vital signs sets (241,996 hospital admissions). Patients were categorised as having no infection, primary infection or secondary infection using ICD-10 codes. NEWS was significantly better at discriminating in-hospital mortality, irrespective of infection status (no infection, NEWS 0.831 (0.825-0.838) vs. qSOFA 0.688 (0.680-0.695); primary infection, NEWS 0.805 (0.799-0.812) vs. qSOFA 0.677 (0.670-0.685)). Similarly, NEWS performed significantly better in all patient groups (all admissions, emergency medicine admissions and emergency surgery admissions) for all outcomes studied. Overall, qSOFA performed no better, and often worse, in admissions with infection than without.

Conclusions: The National Early Warning Score outperforms the quick Sequential [Sepsis-Related] Organ Failure Assessment score, irrespective of infection status. These findings suggest that qSOFA should be re-evaluated as the system of choice for identifying non-ICU patients with suspected infection who are at greater risk of poor outcome. 


\section{INTRODUCTION}

The Sepsis-3 task force recommends the quick Sequential [Sepsis-Related] Organ Failure Assessment (qSOFA) score for identifying patients with suspected infection who are at greater risk of poor outcomes (1). qSOFA assigns one point for each of the following: systolic BP $\leq 100 \mathrm{mmHg}$; respiratory rate $\geq 22$ breaths per min; altered mentation (Table 1) (1). Analysis of a large US database showed an area under the receiver operating characteristic (AUROC) curve for in-hospital mortality of $0.81(95 \% \mathrm{Cl}, 0.80-0.82)$ for patients outside the intensive care unit (ICU) (2).

qSOFA is similar to the early warning scores (EWS) used on general wards to warn that a patient is at high risk of a serious adverse outcome, irrespective of underlying diagnosis (3-5). The UK's National Early Warning Score (NEWS) (6) allocates 0-3 points to each of seven clinical parameters (Table 1). NEWS performs well in a range of clinical settings in different countries (7-17) and is increasingly used as a frontline risk tool.

Introducing qSOFA to settings already using NEWS presents difficulties, as they include similar physiological variables but different weighting thresholds. Operating both is likely to introduce unnecessary duplication of staff effort, needless protocol complexity, confusion with respect to clinical practice and increased educational needs.

Recent publications demonstrate that, in patients with suspected infection, NEWS discriminates in-hospital mortality, ICU admission, and their combined outcome as well as or better than qSOFA $(10,18,19)$. However, there is no published comparison in unselected hospital admissions to inform of a benefit to introducing qSOFA when NEWS is already used. Therefore, we compared the diagnostic performance of qSOFA and NEWS in non-ICU patients with and without a diagnosis of infection (hereafter termed "infection status") during their hospital stay, using a large database of routinely collected vital signs. We used a primary outcome of in-hospital death and four clinically relevant, secondary outcomes. To investigate the impact of reducing NEWS to the same three parameters in qSOFA, we also evaluated the performance of a simplified novel modification of NEWS - quick NEWS (qNEWS) (Table 1).

\section{METHODS}


This retrospective study falls within local research ethics committee approval (08/02/1394) from the Isle of Wight, Portsmouth and South East Hampshire Research Ethics Committee.

\section{Vital Signs database and its development}

A database was created of vital signs collected from consecutive patients aged $\geq 16$ years admitted to a large UK hospital (http://www.porthosp.nhs.uk/about-us) on or after $1^{\text {st }}$ January 2010 and discharged before $1^{\text {st }}$ February 2016. Patients transferred directly on admission to critical care areas were excluded. Similarly, we excluded patients who (a) were discharged alive on the day of admission, or (b) had no observation set recorded in the 24 hours before discharge.

Vital signs were documented at the bedside in real-time using handheld devices running Vitalpac $^{\mathrm{TM}}$ software (The Learning Clinic, London) (20). Vitalpac ${ }^{\mathrm{TM}}$ was used throughout the general wards, but not in the ICU. Each vital signs observation set contained the necessary data items to calculate NEWS and qNEWS values. However, because qSOFA evaluates mental status using the Glasgow Coma Scale (GCS) (1) and the study hospital uses the AVPU scale ((alert (A); responds to voice $(\mathrm{V})$; responds to pain $(\mathrm{P})$; or unresponsive $(\mathrm{U}))$, we categorised patients scoring $\mathrm{V}, \mathrm{P}$ or $\mathrm{U}$ as having 'altered mental status' when calculating qSOFA scores. Vital signs sets for which any measurements were absent were excluded because a full set is required to calculate a NEWS value.

\section{qSOFA, NEWS, qNEWS}

qSOFA and NEWS values were calculated for each vital signs set using the weightings described previously $(1,6)$ (Table 1). We also calculated a 'quick NEWS' (qNEWS) value using only the systolic blood pressure, respiratory rate and AVPU components of NEWS, and the same weightings for each cutoff as in NEWS (Table 1). 


\section{Categorisation of admissions:}

The categorisation of admissions into surgical and medical groups followed a previous method (9) and is described in Supplementary Table 1. Admissions were further categorized as "elective" or "nonelective", as recorded in the hospital's patient administration system (PAS). We analysed three datasets: (a) all admissions, (b) non-elective admissions to surgery, and (c) non-elective admissions to medicine.

\section{Patient comorbidity}

Patient comorbidity on admission was measured using Dr. Foster Intelligence's modification of the Charlson Comorbidity index, as used by the National Health Service (NHS) (21).

\section{Categorisation of presence of infection:}

Patients' infection status was assigned according to the presence/absence of Suspicion of Sepsis (SOS) ICD-10 codes relating to bacterial infection (22). During hospital admission, if a patient is transferred from one specialty to another, or from one consultant to another, a new 'finished consultant episode' (FCE) is generated. An admission has at least one FCE, but may have many. In our database, each FCE can contain up to eighteen diagnoses. Diagnosis one is the primary diagnosis. After inspection of the data and noting that diagnosis two appeared to have been used for subsidiary primary diagnoses, we decided to interpret it thus. Diagnoses three and higher (if present) are secondary diagnoses. Patients were considered to have had a primary infection if either diagnosis one or two of any FCEs contained an SOS ICD-10 code. Patients were considered to have a secondary infection if there was no primary infection but any of the secondary diagnoses of any FCEs contained an SOS ICD-10 code. Admissions with no associated SOS code were categorised to the "No Infection" group.

\section{Outcomes}

We studied a primary outcome of in-hospital death and four secondary outcomes: in-hospital death within 24 hours of a vital signs set; in-hospital death or ICU stay $\geq 3$ days; in-hospital death or ICU admission from a ward; and 'in-hospital death or ICU admission from a ward' within 24 hours of a vital signs set. Outcome data were identified from the hospital PAS and ICU database. Where relevant, the maximum length of ICU stay (to account for multiple admissions in a single hospital stay) was calculated. 


\section{Observation selection methods}

Following a previous method (23), we constructed 10,000 random samples containing one vital signs observation set per admission. The observations were chosen by first randomly selecting a time during every admission, and then choosing the observation set closest to it. Vitalpac ${ }^{\mathrm{TM}}$ time-stamps observations automatically as they are entered at the bedside. We used the first and last observation dates and times to determine the time period from which to choose observations. To avoid biasing against selection of the first and last observation sets, we added to the beginning and end of the selection period a length of time equal to half of the mean time between observation sets for that patient episode. We truncated the analysis at the first ICU stay to avoid the inclusion of vital signs taken after ICU admission.

\section{Statistical analysis}

All statistical analyses were undertaken using R v3.4 (R Core Team) (24). The ability of NEWS, qSOFA and qNEWS to discriminate outcomes were assessed using AUROC analyses. For each scoring system, the AUROC was calculated using the mean AUROC of the 10,000 samples; $95 \%$ confidence intervals were calculated from the distribution. 


\section{RESULTS}

\section{Characteristics of admissions}

There were 751,804 patient admissions in the study period. After exclusions, (Figure 1) 241,966 admissions and 5,435,344 complete vital signs sets were studied. Only 59,300 of 6,298,191 (0.94\%) sets were excluded because they were incomplete (Figure 1). Of the 241,996 admissions, 114,822 were nonelective medical and 47,592 non-elective surgical admissions. Patient demographics are shown in Table 2.

Of the all admissions group, 6798 (2.81\%) were followed by in-hospital death, 2054 (0.85\%) by ICU admission from the wards and $971(0.40 \%)$ by ICU stays $\geq 3 \mathrm{~d}$. There were more in-hospital deaths, ICU admissions and ICU stays $\geq 3 \mathrm{~d}$ for admissions with infection than for those without (odds ratios ranged from 1.75 to 15.55 ) Table 2 . The 25 most common SOS diagnoses and 25 most common nonSOS diagnoses in the dataset, are shown in Supplementary Tables 2 and 3, respectively.

During admission, a total of 44647 (18.5\%) of all patient admissions had a primary and 21536 $(8.9 \%)$ a secondary infection (Table 2). Similar proportions were seen for non-elective medical $(24.3 \%$ and $11.9 \%)$ and non-elective surgical admission subgroups (22.8\% and $8.0 \%)$.

The distribution of patients by age and infection status is shown in Supplementary Figure 1. Generally, the risk of having an infection - either primary or secondary - increased with patient age.

\section{Relationship between infection and scores}

The distributions of qSOFA, NEWS and qNEWS values, and observed risk of in-hospital death by infection status are shown in Supplementary Figures 2a-c, respectively. For each of qSOFA, NEWS and qNEWS, in-hospital mortality increased with rising score value, irrespective of infection status. However, in-hospital mortality was significantly lower for admissions without infection for scores $\leq 3$ (qSOFA), $\leq 7$ (NEWS) and $\leq 4$ (qNEWS). The relationships of qSOFA, NEWS and qNEWS values against the outcome of in-hospital death within 24 hours of a vital signs set are shown in Supplementary Figures 3a-c.

Plots of qSOFA, NEWS and qNEWS values against the percentage of patient admissions with 
each infection status are shown in Supplementary Figures 4a-c. In general, for all three scores, an increasing score value was associated with a greater likelihood of a primary infection. A similar trend was seen for secondary infections. Observation sets with a qSOFA value between 1 and 2 , NEWS value $=5$, and qNEWS value $=4$ were each associated with an approximately $50 \%$ risk of having an infection.

\section{Performance of NEWS, qNEWS and qSOFA}

The AUROC values for NEWS, qNEWS and qSOFA for all 241,966 admissions are shown in Table 3, and for non-elective admissions to surgery and medicine in Table 4. Pictorial displays of the AUROC values for all scores and all outcomes are shown in Supplementary Figures 5a-c for three groups - all admissions (Figure 5a), non-elective admissions to medicine (Figure 5b) and non-elective admissions to surgery $(5 \mathrm{c})$. NEWS performed significantly better than qSOFA for all three patient groups and for all outcomes, irrespective of infection status. Considering the all admissions group and the primary outcome of in-hospital mortality, the AUROC (Cl) value for NEWS was significantly higher $(0.825(0.821-0.829))$ than for qSOFA $(0.681(0.676-0.686))$.

For the all admissions group and primary outcome, qSOFA performed similarly in the no infection and primary infection groups (no infection, 0.688 (0.680-0.695); primary infection, $0.677(0.670-0.685)$ ), whereas NEWS performed better in the no infection group (no infection, 0.831 (0.825-0.838); primary infection, 0.805 (0.799-0.812)) (Table 3). Overall, qSOFA performed no better, and often worse, in admissions with infection than without.

Considering the all admissions group and the primary outcome, sensitivity $(\mathrm{Cl})$ was significantly higher for NEWS values of 5,6 and 7 (46.6\% (45.8-47.4\%), 36.2\% (35.4-37.0\%) and 27.1\% (26.4$27.9 \%)$ ), respectively) than for qSOFA values of $>2(12.5 \%(12.0-13.1 \%))$, but specificity $(\mathrm{Cl})$ was lower (NEWS 5 (95.9\% (95.9-96.0\%)), 6 (98.0 (98.0-98.1\%), 7 (99.1 (99.1-99.1\%)); qSOFA >2 (99.7 (99.7$99.7 \%))$. This relationship was unaltered by infection status.

NEWS consistently showed superior discrimination than qNEWS. However, overall, the performance of qNEWS was significantly better or the same as qSOFA across all groups and outcomes. 
Considering the all admissions group and the primary outcome, the AUROC value for qNEWS was higher than for qSOFA (0.701 (0.696-0.706) vs. $0.681(0.676-0.686))$.

To demonstrate the relationship between sensitivity against trigger rate (i.e., percentage of observations at, or above, a given score value) we plotted an EWS efficiency curve (25) for qSOFA and NEWS. We chose to compare qSOFA and NEWS for admissions with a SOS diagnosis using perhaps the most clinically useful of the outcomes we studied - in-hospital death within 24 hours of a vital signs set. The closer the efficiency curve is to the lower right corner, the higher the efficiency of the EWS (i.e., more outcomes are detected for a lower trigger rate). Supplementary Figure 6 shows that NEWS values of 5 and 7 are more efficient than a qSOFA score $=2$. 


\section{DISCUSSION}

This study compared the ability of qSOFA and NEWS to discriminate in-hospital mortality and four other outcomes in admissions to non-ICU areas of a hospital. Irrespective of infection status, NEWS discriminated all outcomes significantly better than qSOFA. Sensitivity for NEWS values of 5-7 was significantly higher than for qSOFA values of $\geq 2$. Overall, qSOFA performed no better, and often worse, in admissions with infection than without. Even when NEWS was reduced to a version containing the same physiological variables as qSOFA - qNEWS - it performed significantly better or at least as well as qSOFA across all groups and outcomes.

The Sepsis-3 task force reported an AUROC value of 0.81 for qSOFA for mortality in non-ICU patients with suspected infection (2). Others have since described varying qSOFA performance in patients with suspected infection, with poor sensitivity for a range of outcomes being frequently observed $(10,18,19,26-36)$. Two other studies demonstrate that qSOFA performs similarly in patients in whom infection has not been diagnosed or is not suspected, implying that qSOFA is not infection-specific and should simply be regarded as a general EWS $(36,37)$.

In a few studies, qSOFA's performance has been compared with that of other EWS systems, e.g. the modified early warning score (MEWS) and NEWS, but only in patients with suspected or confirmed infection $(10,18,38)$. Innocenti et al. found that MEWS and qSOFA discriminated 28-day mortality at ED admission similarly (38). However, Churpek et al. (10) reported that both NEWS and MEWS were "...more accurate than qSOFA for predicting in-hospital mortality and ICU transfer...", with NEWS also performing significantly better than MEWS for in-hospital mortality (10). These relationships appear to be constant, irrespective of the criteria used to define 'suspicion of infection' (18).

That qSOFA performs similarly in patients with and without a primary infection is not surprising, as the abnormalities that cause raised qSOFA scores (and NEWS values) in infection also occur in ischemia, inflammation and trauma (39). That NEWS performs better than qSOFA, irrespective of the presence/absence of infection, should also be expected as NEWS contains additional parameters known to be valuable in identifying high-risk patients. 
NEWS and qSOFA do not diagnose infection; they merely identify patients with a high risk of adverse outcomes. In this respect, NEWS has already shown superior discrimination to other EWS systems $(7,8,10)$ and the physiological components of Medical Emergency Team calling criteria (40). The current study now confirms NEWS's superiority over qSOFA. Consequently, there seems to be no benefit for hospitals already using NEWS to change to or introduce qSOFA. NEWS is already the recommended EWS for the UK (6), with the NHS (41), the Joint Royal Colleges Ambulance Liaison Committee and the UK Association of Ambulance Chief Executives (42) highlighting its use in the suspicion and management of sepsis. The current study results suggest that the Sepsis-3 task force should re-evaluate their recommendation to use qSOFA and reflect upon whether NEWS is a more appropriate score to use, even when infection is suspected.

This study has several strengths. It is a large multi-year study, with 241,966 completed admissions to a large general hospital. It studies over 5.4 million complete vital signs sets, each recorded in a standard manner as part of routine care using identical electronic devices (20). It is the first to compare the performance of qSOFA and NEWS in general ward patients with/without an infection diagnosis. It uses common, standardized, systems for measuring patient comorbidity (21) and categorising infection (22).

The study also has limitations. Whilst each vital signs set contained the data necessary to calculate a NEWS value, GCS score values were not available to calculate a qSOFA score and AVPU was used. This may have disadvantaged qSOFA compared to NEWS, as GCS is likely to be more sensitive than AVPU for mild altered mental status. We obtained the date/time of death/discharge from the hospital's PAS and these data are likely to be systematically late, such that the number of observations followed by death within 24 hours may have been underestimated.

We were unable to study data from the ED as these were unavailable; the operating characteristics of NEWS and qSOFA in the ED could vary from that obtained in other areas of the hospital. However, we undertook a post-hoc analysis comparing the performance of qSOFA and NEWS for two admission groups: (a) those admitted from the ED to non-ICU ward areas of the hospital $(n=$ $121,952)$, and (b) those admitted directly to non-ICU ward areas of the hospital, without attending the ED 
$(n=120,044)$ (Supplementary Table 4). The results are similar for the two groups suggesting that the route of admission is immaterial to the operating characteristics of NEWS and qSOFA.

Patients on an end-of-life (EoL) pathway could not be explicitly excluded, although we partially mitigated this by excluding those with no complete vital signs sets in the final 24 hours of their stay (it is hospital policy to cease the collection of complete sets of vital signs once a patient enters the EoL pathway). As it would not necessarily be anticipated that a patient would be subsequently managed on an EoL pathway at the time an EWS is applied, we repeated the analyses without excluding admissions where vital signs were not measured in the 24 hours prior to discharge (Supplementary Table 5). We included patients with Do Not Attempt Cardiopulmonary Resuscitation decisions, as such patients continue to receive normal care, including the measurement of vital signs. Finally, the study was conducted in a single site where the precursor of NEWS - ViEWS (25) - was developed and requires validation in independent data sets in various settings.

\section{Conclusions}

In this single centre study, NEWS was a significantly better discriminator than qSOFA for identifying non-ICU patients at greater risk of poor outcomes, irrespective of infection status. Overall, qSOFA performed no better and often worse in admissions with infection than without, suggesting that qSOFA should be regarded as no more than another non-specific, but poorly performing, EWS. For hospitals already using NEWS, there seem to be no benefit to changing to or adding qSOFA, even when infection is suspected. The Sepsis-3 task force recommendation to use qSOFA as the system of choice for identifying patients with suspected infection at greater risk of poor outcomes requires re-evaluation. Hospitals seeking a high-performing EWS for identifying patients at high risk of adverse outcomes from any underlying condition, including infection, should consider introducing NEWS. 
LEGENDS FOR FIGURES

Figure 1

Data flow diagram for the study. 


\section{REFERENCES}

1. Singer M, Deutschman CS, Seymour CW, et al. The Third International Consensus Definitions for Sepsis and Septic Shock (Sepsis-3). JAMA 2016;315:801-810. doi:10.1001/jama.2016.0287

2. Seymour CW, Liu VX, Iwashyna TJ, et al. Assessment of clinical criteria for sepsis: for the Third International Consensus Definitions for Sepsis and Septic Shock (Sepsis-3). JAMA 2016;315:762774. doi:10.1001/jama.2016.0288

3. Subbe CP, Kruger M, Rutherford P, Gemmel L. Validation of a modified early warning score in medical admissions. QJM 2001;94:521-526.

4. Smith GB, Prytherch DR, Schmidt P, Featherstone PI. Review and performance evaluation of aggregate weighted 'track and trigger' systems. Resuscitation 2008;77:170-9. doi: 10.1016/j.resuscitation.2007.12.004.1

5. Smith ME, Chiovaro JC, O'Neil M, et al. Early warning system scores for clinical deterioration in hospitalized patients: a systematic review. Ann Am Thorac Soc 2014;11:1454-65. doi: 10.1513/AnnalsATS.201403-102OC.

6. Royal College of Physicians of London. National Early Warning Score (NEWS): Standardising the assessment of acute illness severity in the NHS. Report of a working party. London; 2012.

7. Smith GB, Prytherch DR, Meredith P, Schmidt P, Featherstone PI. The ability of the National Early Warning Score (NEWS) to discriminate patients at risk of early cardiac arrest, unanticipated intensive care unit admission, and death. Resuscitation 2013;4:465-470. doi: 10.1016/j.resuscitation.2012.12.016.

8. Abbott TE, Vaid N, Ip D, et al. A single-centre observational cohort study of admission National Early Warning Score (NEWS). Resuscitation 2015;92:89-93. doi: 10.1016/j.resuscitation.2015.04.020.

9. Kovacs C, Jarvis SW, Prytherch DR, et al. Comparison of the National Early Warning Score in nonelective medical and surgical patients. Br J Surg 2016;103:1385-1393. doi: 10.1002/bjs.10267.

10. Churpek MM, Snyder A, Han X, et al: qSOFA, SIRS, and early warning scores for detecting clinical deterioration in infected patients outside the ICU. Am J Respir Crit Care Med 2017;195:906-911. doi: 10.1164/rccm.201604-08540C.

11. Roberts R, Djärv T. Preceding national early warnings scores among in-hospital cardiac arrests and their impact on survival. Am J Emerg Med 2017; pii: S0735-6757(17)30359-5. doi: 10.1016/j.ajem.2017.04.072. [Epub ahead of print]. 
12. Shaw J, Fothergill RT, Clark S, Moore F. Can the prehospital National Early Warning Score identify patients most at risk from subsequent deterioration? Emerg Med J 2017 Aug;34:533-537. doi: 10.1136/emermed-2016-206115.

13. Spångfors M, Arvidsson L, Karlsson V, Samuelson K. The National Early Warning Score: Translation, testing and prediction in a Swedish setting. Intensive Crit Care Nurs 2016;37:62-67. doi: 10.1016/j.iccn.2016.05.007.

14. Spagnolli W, Rigoni M, Torri E, Cozzio S, Vettorato E, Nollo G. Application of the National Early Warning Score (NEWS) as a stratification tool on admission in an Italian acute medical ward: A perspective study. Int J Clin Pract 2017 Mar;71(3-4). doi: 10.1111/ijcp.12934.

15. Alam N, Vegting IL, Houben E, et al. Exploring the performance of the National Early Warning Score (NEWS) in a European emergency department. Resuscitation 2015;90:111-115. doi: 10.1016/j.resuscitation.2015.02.011.

16. Silcock DJ, Corfield AR, Gowens PA, Rooney KD. Validation of the National Early Warning Score in the prehospital setting. Resuscitation 2015;89:31-35.

17. Bilben B, Grandal L, Søvik S. National Early Warning Score (NEWS) as an emergency department predictor of disease severity and 90-day survival in the acutely dyspneic patient - a prospective observational study. Scand J Trauma Resusc Emerg Med 2016;24:80 doi: 10.1186/s13049-0160273-9.

18. Churpek, MM, Snyder A, Sokol S, Pettit NN, Edelson DP. Investigating the Impact of Different Suspicion of Infection Criteria on the Accuracy of Quick Sepsis-Related Organ Failure Assessment, Systemic Inflammatory Response Syndrome, and Early Warning Scores. Crit Care Med 2017 Jul 21. doi: $10.1097 / C C M .0000000000002648$.

19. Goulden R, Hoyle M-C, Monis J, et al. qSOFA, SIRS and NEWS for predicting inhospital mortality and ICU admission in emergency admissions treated as sepsis. Emerg Med J 2018;0:1-5. doi:10.1136/emermed-2017-207120.

20. Smith GB, Prytherch DR, Schmidt P, et al. Hospital-wide physiological surveillance - a new approach to the early identification and management of the sick patient. Resuscitation 2006; 71:1928.

21. Dr Foster Intelligence. Understanding HSMRs. A Toolkit on Hospital Standardised Mortality Ratios. Version 9: July 2014. London; 2014. 
22. Inada-Kim M, Page B, Maqsood I, Vincent C. Defining and measuring suspicion of sepsis: an analysis of routine data. BMJ Open 2017; 7:e014885. doi: 10.1136/bmjopen-2016-014885.

23. Jarvis SW, Kovacs C, Briggs $\mathrm{J}$, et al. Are observation selection methods important when comparing early warning score performance? Resuscitation 2015; 90:1-6. doi:

10.1016/j.resuscitation.2015.01.033.

24. R Development Core Team. R: A Language and Environment for Statistical Computing. R Foundation for Statistical Computing: Vienna, 2014.

25. Prytherch DR, Smith GB, Schmidt PE, Featherstone PI. ViEWS - towards a national early warning score for detecting adult inpatient deterioration. Resuscitation 2010; 81:932-937. doi: 10.1016/j.resuscitation.2010.04.014.

26. Freund Y, Lemachatti N, Krastinova E, et al. Prognostic Accuracy of Sepsis-3 Criteria for In-Hospital Mortality Among Patients With Suspected Infection Presenting to the Emergency Department. JAMA 2017; 317:301-308. doi: 10.1001/jama.2016.20329.

27. Kim M, Ahn S, Kim WY, et al. Predictive performance of the quick Sequential Organ Failure Assessment score as a screening tool for sepsis, mortality, and intensive care unit admission in patients with febrile neutropenia. Support Care Cancer 2017; 25:1557-1562. doi: 10.1007/s00520016-3567-6.

28. Mellhammar L, Wullt S, Lindberg A, Lanbeck P, Christensson B, Linder A. Sepsis Incidence: A Population-Based Study. Open Forum Infectious Diseases 2016; 3:ofw207.

29. Giamarellos-Bourboulis EJ, Tsaganos T, Tsangaris I, et al. Validation of the new Sepsis-3 definitions: proposal for improvement in early risk identification. Clin Microbiol Infect 2017; 23:104-109. doi: 10.1016/j.cmi.2016.11.003.

30. Dorsett M, Kroll M, Smith CS, Asaro P, Liang SY, Moy HP. qSOFA Has Poor Sensitivity for Prehospital Identification of Severe Sepsis and Septic Shock. Prehosp Emerg Care 2017; 21:489497. doi: 10.1080/10903127.2016.1274348.

31. Williams JM, Greenslade JH, McKenzie JV, Chu K, Brown AF, Lipman J. Systemic Inflammatory Response Syndrome, Quick Sequential Organ Function Assessment, and Organ Dysfunction: Insights From a Prospective Database of ED Patients With Infection. Chest 2017; 151:586-596. doi: 10.1016/j.chest.2016.10.057. 
32. Askim A, Moser F, Gustad LT, et al. Poor performance of quick-SOFA (qSOFA) score in predicting severe sepsis and mortality - a prospective study of patients admitted with infection to the emergency department. Scand J Trauma Resusc Emerg Med 2017; 25:56. doi: 10.1186/s13049-017-0399-4.

33. Haydar S, Spanier M, Weems P, Wood S, Strout T. Comparison of qSOFA score and SIRS criteria as screening mechanisms for emergency department sepsis. Am J Emerg Med 2017 Jul 6. pii: S0735-6757(17)30509-0. doi: 10.1016/j.ajem.2017.07.001. [Epub ahead of print]

34. Moskowitz A, Patel PV, Grossestreuer AV, et al. Quick Sequential Organ Failure Assessment and Systemic Inflammatory Response Syndrome Criteria as Predictors of Critical Care Intervention Among Patients With Suspected Infection. Crit Care Med 2017 Jul 28. doi:

10.1097/CCM.0000000000002622. [Epub ahead of print]

35. Park HK, Kim WY, Kim MC, Jung W, Ko BS. Quick sequential organ failure assessment compared to systemic inflammatory response syndrome for predicting sepsis in emergency department. $J$ Crit Care 2017; 42:12-17. doi: 10.1016/j.jcrc.2017.06.020.

36. Brabrand M, Havshoj U, Graham CA. Validation of the qSOFA score for identification of septic patients: A retrospective study. Eur J Intern Med 2016; 36:e35-e36. doi: 10.1016/j.ejim.2016.09.004.

37. Singer AJ, Ng J, Thode HC, Jr., Spiegel R, Weingart S. Quick SOFA Scores Predict Mortality in Adult Emergency Department Patients With and Without Suspected Infection. Ann Emerg Med 2017;69:475-479. doi: 10.1016/j.annemergmed.2016.10.007.

38. Innocenti F, Tozzi C, Donnini C, et al. SOFA score in septic patients: incremental prognostic value over age, comorbidities, and parameters of sepsis severity. Intern Emerg Med 2017 Feb 10. doi: 10.1007/s11739-017-1629-5.

39. Bone RC, Balk RA, Cerra FB, et al. Definition for sepsis and organ failure and guidelines for the use of innovative therapies in sepsis: the American College of Chest Physicians/Society of Critical Care Medicine Consensus Conference Committee. Chest 1992; 101:1644-1655.

40. Smith GB, Prytherch DR, Jarvis SW et al. A comparison of the ability of the physiologic components of Medical Emergency Team criteria and the UK National Early Warning Score to discriminate patients at risk of a range of adverse clinical outcomes. Crit Care Med 2016; 44:2171-2181.

41. NHS England. Sepsis guidance implementation advice for adults. London 2017.

42. Joint Royal Colleges Ambulance Liaison Committee and Association of Ambulance Chief Executives. JRCALC Clinical Practice Supplementary Guidelines 2017. London, 2017. 
Figure 1

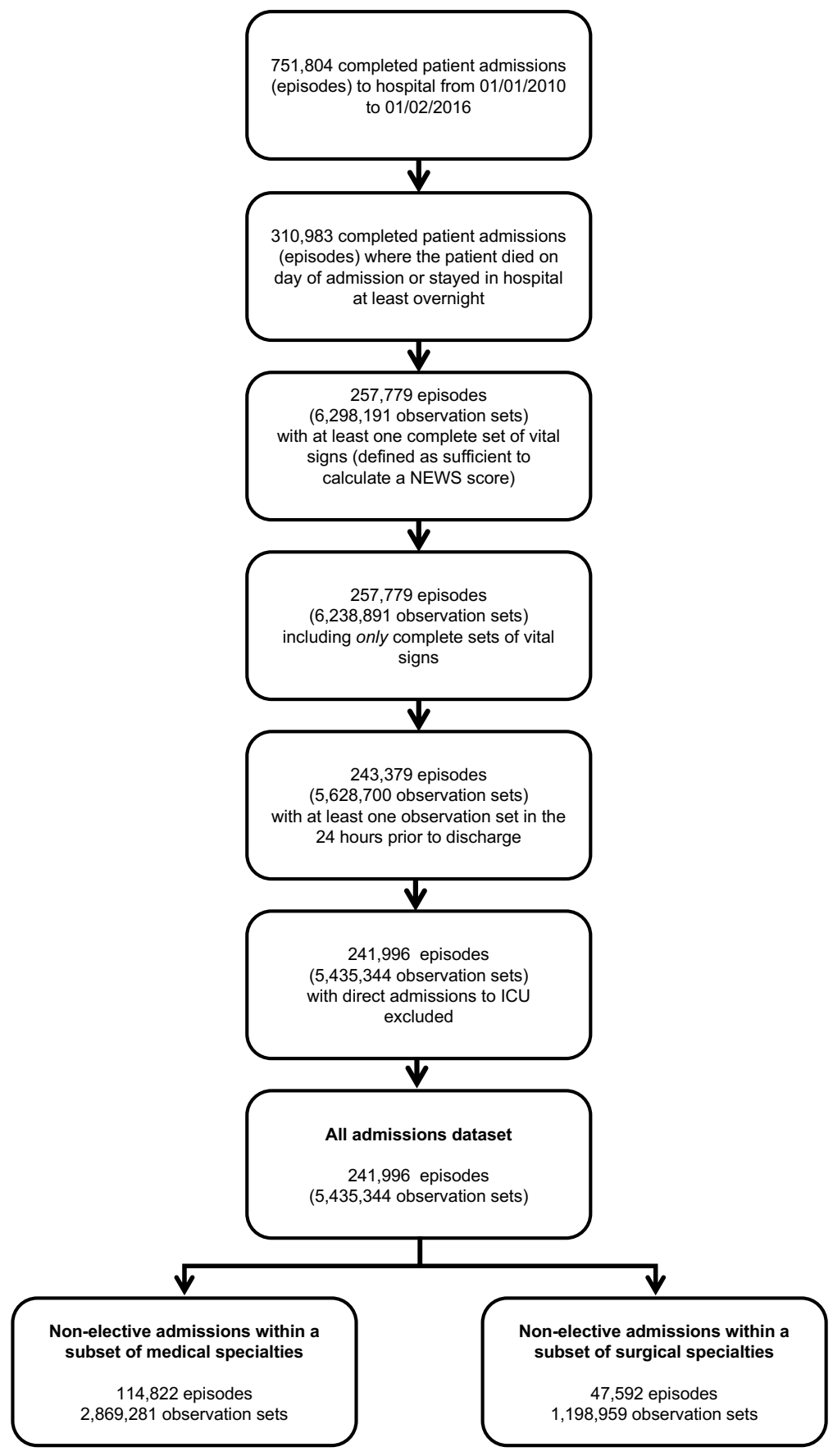


Table 1: Scoring systems evaluated in the study and their weightings

\begin{tabular}{|c|c|c|c|c|c|c|c|}
\hline Physiological parameters & 3 & 2 & 1 & 0 & 1 & 2 & 3 \\
\hline \multicolumn{8}{|l|}{$\begin{array}{l}\text { quick Sequential [Sepsis-related] } \\
\text { Organ Failure Assessment (qSOFA) } \\
\text { score }\end{array}$} \\
\hline Respiration Rate (breaths per minute) & & & $\geq 22$ & & & & \\
\hline Systolic BP $(\mathrm{mmHg})$ & & & $\leq 100$ & & & & \\
\hline Altered mentation & & & $\mathrm{GCS}<15$ & & & & \\
\hline & & & & & & & \\
\hline \multicolumn{8}{|l|}{ NEWS } \\
\hline Respiration Rate (breaths per minute) & $\leq 8$ & & $9-11$ & $12-20$ & & $21-24$ & $\geq 25$ \\
\hline $\mathrm{SpO}_{2} \%$ & $\leq 91$ & $92-93$ & $94-95$ & $\geq 96$ & & & \\
\hline Any supplemental oxygen? & & Yes & & No & & & \\
\hline Temperature $\left({ }^{\circ} \mathrm{C}\right)$ & $\leq 35.0$ & & $35.1-36.0$ & $36.1-38.0$ & $38.1-39.0$ & $\geq 39.1$ & \\
\hline Systolic BP (mmHg) & $\leq 90$ & $91-100$ & $101-110$ & $111-219$ & & & $\geq 220$ \\
\hline Heart/pulse rate (beats per minute) & $\leq 40$ & & $41-50$ & $51-90$ & $91-110$ & $111-130$ & $\geq 131$ \\
\hline Level of consciousness (AVPU) & & & & A & & & $\mathrm{V}, \mathrm{P}$ or $\mathrm{U}$ \\
\hline & & & & & & & \\
\hline \multicolumn{8}{|l|}{$\begin{array}{l}\text { quick National Early Warning Score } \\
\text { (qNEWS) }\end{array}$} \\
\hline Respiration Rate (breaths per minute) & $\leq 8$ & & $9-11$ & $12-20$ & & $21-24$ & $\geq 25$ \\
\hline Systolic BP (mmHg) & $\leq 90$ & $91-100$ & $101-110$ & $111-219$ & & & $\geq 220$ \\
\hline Level of consciousness (AVPU) & & & & A & & & $\mathrm{V}, \mathrm{P}$ or $\mathrm{U}$ \\
\hline
\end{tabular}

Level of consciousness: $A=$ Alert; $V=$ Responds to voice; $P=$ Responds to pain; $U$ = Unresponsive GCS: Glasgow Coma Scale score 
Table 2: Patient demographic and observation data regarding admissions

\begin{tabular}{|c|c|c|c|c|c|c|c|}
\hline $\begin{array}{l}\text { Admission } \\
\text { group }\end{array}$ & & $\begin{array}{c}\text { All } \\
\text { Admissions }\end{array}$ & $\begin{array}{c}\text { Non-Elective } \\
\text { Admissions } \\
\text { to } \\
\text { Medical } \\
\text { Specialties }\end{array}$ & $\begin{array}{c}\text { Non-Elective } \\
\text { Admissions } \\
\text { to } \\
\text { Surgical } \\
\text { Specialties }\end{array}$ & $\begin{array}{l}\text { No infection } \\
\text { code }\end{array}$ & $\begin{array}{l}\text { Primary } \\
\text { infection } \\
\text { code }\end{array}$ & $\begin{array}{l}\text { Secondary } \\
\text { infection } \\
\text { code }\end{array}$ \\
\hline \multirow[t]{6}{*}{ All } & Number of admissions; ( $\%$ Male) & 241996; (47.0) & & & $175813 ;(47.2)$ & $44647 ;(47.5)$ & 21536; (44.5) \\
\hline & Age, (mean/median/SD) & $63 / 67 / 20$ & & & $62 / 65 / 20$ & $65 / 70 / 22$ & $72 / 77 / 18$ \\
\hline & Death in-hospital, n (\%) & $6798(2.81)$ & & & $2362(1.34)$ & $3035(6.8)^{\mathrm{a}}$ & $1401(6.51)^{b}$ \\
\hline & ICU admission, $\mathrm{n}(\%)$ & $2054(0.849)$ & & & $665(0.378)$ & $740(1.66)^{\mathrm{C}}$ & $649(3.01)^{d}$ \\
\hline & ICU stay $\geq 3 \mathrm{~d}, \mathrm{n}(\%)$ & $971(0.401)$ & & & $192(0.109)$ & $419(0.938)^{\mathrm{e}}$ & $360(1.67)^{f}$ \\
\hline & Comorbidity index (IQR) & $0(9)$ & & & $0(7)$ & $4(13)$ & $5(14)$ \\
\hline \multirow[t]{13}{*}{ Surgical } & Number of admissions; (\% Male) & & & $47592 ;(47.0)$ & $32902 ;(47.3)$ & $10874 ;(47.0)$ & $3816 ;(45.0)$ \\
\hline & Age, (mean/median/SD) & & & $57 / 59 / 22$ & $58 / 60 / 22$ & $50 / 48 / 21$ & $71 / 76 / 19$ \\
\hline & Death in-hospital, n (\%) & & & $863(1.81)$ & $413(1.26)$ & $237(2.18)^{g}$ & $213(5.58)^{h}$ \\
\hline & ICU admission, $\mathrm{n}(\%)$ & & & $764(1.61)$ & $271(0.824)$ & $238(2.19)^{j}$ & $255(6.68)^{\mathrm{k}}$ \\
\hline & ICU stay $\geq 3 d, n(\%)$ & & & $319(0.67)$ & $76(0.231)$ & $122(1.12)^{\mathrm{m}}$ & $121(3.17)^{n}$ \\
\hline & Comorbidity index (IQR) & & & $0(4)$ & $0(4)$ & $0(4)$ & $4(14)$ \\
\hline & General Surgery, n & & & 11029 & 7236 & 3082 & 711 \\
\hline & Urology, $\mathrm{n}$ & & & 6129 & 4016 & 1643 & 470 \\
\hline & Renal Surgery \& Transplantation, $\mathrm{n}$ & & & 644 & 509 & 58 & 77 \\
\hline & Colorectal, n & & & 11618 & 7580 & 3379 & 659 \\
\hline & Upper Gastrointestinal, $\mathrm{n}$ & & & 5110 & 3338 & 1502 & 270 \\
\hline & Vascular, $\mathrm{n}$ & & & 520 & 320 & 68 & 132 \\
\hline & Trauma \& Orthopaedics & & & 12542 & 9903 & 1142 & 1497 \\
\hline \multirow[t]{6}{*}{ Medical } & Number of admissions; (\% Male) & & 114822; (49.2) & & $73198 ;(50.2)$ & $27932 ;(48.9)$ & 13692; (44.1) \\
\hline & Age, (mean/median/SD) & & $69 / 73 / 19$ & & $66 / 69 / 19$ & $73 / 77 / 17$ & $74 / 78 / 17$ \\
\hline & Death in-hospital, n (\%) & & $5404(4.71)$ & & $1703(2.33)$ & $2660(9.52)^{p}$ & $1041(7.6)^{\mathrm{q}}$ \\
\hline & ICU admission, $\mathrm{n}(\%)$ & & $896(0.78)$ & & $243(0.332)$ & $412(1.48)^{r}$ & $241(1.76)^{s}$ \\
\hline & ICU stay $\geq 3 \mathrm{~d}, \mathrm{n}(\%)$ & & $467(0.407)$ & & $75(0.102)$ & $246(0.881)^{t}$ & $146(1.07)^{\mathrm{u}}$ \\
\hline & Comorbidity index (IQR) & & $4(13)$ & & $4(11)$ & $8(15)$ & $8(16)$ \\
\hline
\end{tabular}




\begin{tabular}{|c|c|c|c|c|}
\hline General Medicine, $\mathrm{n}$ & 89108 & 55590 & 23367 & 10151 \\
\hline Gastroenterology, $\mathrm{n}$ & 929 & 749 & 90 & 90 \\
\hline Endocrinology, $\mathrm{n}$ & 302 & 223 & 35 & 44 \\
\hline Clinical Haematology, $\mathrm{n}$ & 1495 & 812 & 380 & 303 \\
\hline Rehabilitation, $\mathrm{n}$ & 24 & 16 & 0 & 8 \\
\hline Cardiology, $\mathrm{n}$ & 7520 & 6711 & 231 & 578 \\
\hline Respiratory (Thoracic) Medicine, $\mathrm{n}$ & 2960 & 1396 & 1172 & 392 \\
\hline Nephrology, $\mathrm{n}$ & 6280 & 3449 & 1588 & 1243 \\
\hline Medical Oncology, $\mathrm{n}$ & 2830 & 1854 & 594 & 382 \\
\hline Neurology, $\mathrm{n}$ & 6 & 4 & 0 & 2 \\
\hline Rheumatology, $\mathrm{n}$ & 177 & 127 & 32 & 18 \\
\hline Geriatric Medicine, $\mathrm{n}$ & 3191 & 2267 & 443 & 481 \\
\hline
\end{tabular}

a Odds ratio for primary infection vs. no infection $=5.36(5.07-5.66)$

${ }^{b}$ Odds ratio for secondary infection vs. no infection $=5.11(4.77-5.47)$

${ }^{c}$ Odds ratio for primary infection vs. no infection $=4.44(3.99-4.94)$

$\mathrm{d}$ Odds ratio for secondary infection vs. no infection $=8.18(7.33-9.14)$

e Odds ratio for primary infection vs. no infection $=8.67(7.29-10.34)$

${ }^{f}$ Odds ratio for secondary infection vs. no infection $=15.55(13.01-18.64)$

g Odds ratio for primary infection vs. no infection $=1.75(1.49-2.06)$

h Odds ratio for secondary infection vs. no infection $=4.65(3.91-5.52)$

j Odds ratio for primary infection vs. no infection $=2.69(2.25-3.22)$
${ }^{k}$ Odds ratio for secondary infection vs. no infection $=8.62(7.21-10.31)$

$\mathrm{m}$ Odds ratio for primary infection vs. no infection $=4.9$ (3.65-6.62)

${ }^{n}$ Odds ratio for secondary infection vs. no infection $=14.14(10.5-19.14)$

$p$ Odds ratio for primary infection vs. no infection $=4.42(4.15-4.71)$

q Odds ratio for secondary infection vs. no infection $=3.45(3.19-3.74)$

$r$ Odds ratio for primary infection vs. no infection $=4.49(3.82-5.29)$

s Odds ratio for secondary infection vs. no infection $=5.38(4.48-6.46)$

${ }^{t}$ Odds ratio for primary infection vs. no infection $=8.66(6.66-11.38)$

u Odds ratio for secondary infection vs. no infection $=10.51(7.9-14.08)$ 
Table 3: Area under the receiver operator characteristics curve for quick Sequential [Sepsis-related] Organ Failure Assessment (qSOFA) score, the National Early Warning Score (NEWS) and the quick National Early Warning Score (qNEWS) for a range of outcomes for the all admissions group.

\begin{tabular}{|c|c|c|c|c|}
\hline All admissions & & qSOFA & NEWS & qNEWS \\
\hline Outcome & & & & \\
\hline \multirow{5}{*}{ In-hospital Mortality } & All admissions & $0.681(0.676-0.686)$ & $0.825(0.821-0.829)$ & $0.701(0.696-0.706)$ \\
\hline & $1^{\text {ry }}$ infection code & $0.677(0.670-0.685)$ & $0.805(0.799-0.812)$ & $0.694(0.686-0.702)$ \\
\hline & $2^{\text {ry }}$ infection code & $0.643(0.632-0.653)$ & $0.759(0.748-0.769)$ & $0.663(0.651-0.675)$ \\
\hline & $1^{\text {ry }}$ or $2^{\text {ry }}$ infection code & $0.666(0.660-0.672)$ & $0.790(0.785-0.796)$ & $0.684(0.677-0.691)$ \\
\hline & No infection code & $0.688(0.680-0.695)$ & $0.831(0.825-0.838)$ & $0.711(0.703-0.719)$ \\
\hline \multirow{5}{*}{ In-hospital Mortality or ICU $\geq 3$ days } & All admissions & $0.678(0.674-0.683)$ & $0.824(0.820-0.828)$ & $0.698(0.693-0.703)$ \\
\hline & $1^{\text {ry }}$ infection code & $0.680(0.674-0.687)$ & $0.810(0.804-0.816)$ & $0.698(0.690-0.705)$ \\
\hline & $2^{\text {ry }}$ infection code & $0.637(0.628-0.647)$ & $0.758(0.749-0.767)$ & $0.657(0.646-0.667)$ \\
\hline & $1^{\text {ry }}$ or $2^{\text {ry }}$ infection code & $0.666(0.660-0.671)$ & $0.792(0.787-0.797)$ & $0.683(0.677-0.690)$ \\
\hline & No infection code & $0.683(0.676-0.690)$ & $0.826(0.820-0.833)$ & $0.706(0.698-0.714)$ \\
\hline \multirow{5}{*}{ Mortality within 24 hours } & All admissions & $0.798(0.790-0.805)$ & $0.918(0.913-0.923)$ & $0.823(0.815-0.831)$ \\
\hline & $1^{\text {ry }}$ infection code & $0.811(0.798-0.823)$ & $0.919(0.911-0.926)$ & $0.834(0.821-0.847)$ \\
\hline & $2^{\text {ry }}$ infection code & $0.789(0.768-0.811)$ & $0.906(0.891-0.920)$ & $0.815(0.794-0.837)$ \\
\hline & $1^{\text {ry }}$ or $2^{\text {ry }}$ infection code & $0.805(0.795-0.816)$ & $0.916(0.909-0.922)$ & $0.829(0.819-0.841)$ \\
\hline & No infection code & $0.778(0.767-0.789)$ & $0.904(0.896-0.912)$ & $0.805(0.794-0.816)$ \\
\hline \multirow{5}{*}{ In-hospital Mortality or unanticipated ICU admission } & All admissions & $0.671(0.666-0.675)$ & $0.813(0.810-0.817)$ & $0.689(0.685-0.694)$ \\
\hline & $1^{\text {ry }}$ infection code & $0.680(0.673-0.686)$ & $0.808(0.803-0.814)$ & $0.697(0.690-0.704)$ \\
\hline & $2^{\text {ry }}$ infection code & $0.629(0.621-0.638)$ & $0.749(0.741-0.758)$ & $0.647(0.637-0.657)$ \\
\hline & $1^{\text {ry }}$ or $2^{\text {ry }}$ infection code & $0.662(0.657-0.667)$ & $0.787(0.782-0.791)$ & $0.679(0.673-0.685)$ \\
\hline & No infection code & 0.669 (0.663-0.676) & $0.806(0.800-0.812)$ & $0.689(0.682-0.696)$ \\
\hline
\end{tabular}


Table 4: Area under the receiver operator characteristics curve for quick Sequential [Sepsis-related] Organ Failure Assessment (qSOFA) score, the National Early Warning Score (NEWS) and the quick National Early Warning Score (qNEWS) for a range of outcomes.

\begin{tabular}{|c|c|c|c|c|}
\hline \multicolumn{5}{|l|}{ Non-elective admissions to medicine } \\
\hline Outcome & & qSOFA & NEWS & qNEWS \\
\hline \multirow{5}{*}{ In-hospital Mortality } & All admissions & $0.685(0.680-0.690)$ & $0.820(0.815-0.824)$ & $0.710(0.704-0.716)$ \\
\hline & $1^{\text {ry }}$ infection code & $0.679(0.671-0.687)$ & $0.781(0.775-0.788)$ & $0.701(0.693-0.710)$ \\
\hline & $2^{\text {ry }}$ infection code & $0.651(0.639-0.663)$ & $0.766(0.755-0.777)$ & $0.675(0.661-0.688)$ \\
\hline & $1^{\text {ry }}$ or $2^{\text {ry }}$ infection code & $0.671(0.665-0.678)$ & $0.778(0.772-0.784)$ & $0.694(0.687-0.701)$ \\
\hline & No infection code & $0.697(0.688-0.705)$ & $0.839(0.832-0.847)$ & $0.726(0.717-0.735)$ \\
\hline \multirow{5}{*}{ In-hospital Mortality or ICU $\geq 3$ days } & All admissions & $0.686(0.681-0.691)$ & $0.821(0.817-0.825)$ & $0.711(0.706-0.717)$ \\
\hline & $1^{\text {ry }}$ infection code & $0.683(0.676-0.691)$ & $0.788(0.781-0.794)$ & $0.706(0.698-0.714)$ \\
\hline & $2^{\text {ry }}$ infection code & $0.653(0.641-0.664)$ & $0.768(0.757-0.778)$ & $0.675(0.662-0.688)$ \\
\hline & $1^{\text {ry }}$ or $2^{\text {ry }}$ infection code & $0.674(0.668-0.681)$ & $0.782(0.777-0.788)$ & $0.697(0.690-0.704)$ \\
\hline & No infection code & $0.695(0.686-0.704)$ & $0.837(0.829-0.844)$ & $0.724(0.715-0.733)$ \\
\hline \multirow{5}{*}{ Mortality within 24 hours } & All admissions & $0.798(0.790-0.807)$ & $0.911(0.905-0.916)$ & $0.826(0.817-0.834)$ \\
\hline & $1^{\text {ry }}$ infection code & $0.806(0.793-0.819)$ & $0.899(0.890-0.908)$ & $0.829(0.816-0.843)$ \\
\hline & $2^{\text {ry }}$ infection code & $0.785(0.762-0.809)$ & $0.897(0.881-0.912)$ & $0.813(0.789-0.837)$ \\
\hline & $1^{\text {ry }}$ or $2^{\text {ry }}$ infection code & $0.801(0.790-0.812)$ & $0.900(0.892-0.907)$ & $0.826(0.814-0.837)$ \\
\hline & No infection code & $0.784(0.772-0.797)$ & $0.908(0.900-0.917)$ & $0.816(0.803-0.829)$ \\
\hline \multirow{5}{*}{ In-hospital Mortality or unanticipated ICU admission } & All admissions & $0.685(0.680-0.690)$ & $0.818(0.814-0.823)$ & $0.710(0.704-0.715)$ \\
\hline & $1^{\text {ry }}$ infection code & $0.684(0.677-0.691)$ & $0.789(0.783-0.795)$ & $0.707(0.699-0.716)$ \\
\hline & $2^{\text {ry }}$ infection code & $0.649(0.638-0.660)$ & $0.765(0.754-0.775)$ & $0.672(0.659-0.684)$ \\
\hline & $1^{\text {ry }}$ or $2^{\text {ry }}$ infection code & $0.674(0.668-0.680)$ & $0.782(0.777-0.787)$ & $0.697(0.690-0.704)$ \\
\hline & No infection code & $0.691(0.683-0.700)$ & $0.829(0.822-0.836)$ & $0.719(0.711-0.728)$ \\
\hline \multirow{5}{*}{$\begin{array}{r}\text { Mortality within } 24 \text { hours or unanticipated ICU admission } \\
\text { within } 24 \text { hours }\end{array}$} & All admissions & $0.787(0.779-0.794)$ & $0.901(0.895-0.906)$ & $0.814(0.806-0.822)$ \\
\hline & $1^{\text {ry }}$ infection code & $0.796(0.785-0.807)$ & $0.894(0.886-0.902)$ & $0.823(0.811-0.835)$ \\
\hline & $2^{\text {ry }}$ infection code & $0.771(0.750-0.790)$ & $0.884(0.869-0.898)$ & $0.794(0.774-0.815)$ \\
\hline & $1^{\text {ry }}$ or $2^{\text {ry }}$ infection code & $0.790(0.780-0.800)$ & $0.892(0.885-0.899)$ & $0.816(0.805-0.826)$ \\
\hline & No infection code & $0.770(0.758-0.781)$ & $0.890(0.881-0.899)$ & $0.800(0.788-0.812)$ \\
\hline \multicolumn{5}{|l|}{ Non-elective admissions to surgery } \\
\hline OUTCOME & & qSOFA & NEWS & qNEWS \\
\hline In-hospital Mortality & All admissions & $0.647(0.635-0.660)$ & $0.796(0.783-0.808)$ & $0.656(0.641-0.670)$ \\
\hline
\end{tabular}




\begin{tabular}{|c|c|c|c|c|}
\hline & $1^{\text {ry }}$ infection code & $0.668(0.643-0.693)$ & $0.820(0.796-0.843)$ & $0.678(0.650-0.707)$ \\
\hline & $2^{\text {ry }}$ infection code & $0.593(0.566-0.620)$ & $0.717(0.688-0.746)$ & $0.600(0.569-0.631)$ \\
\hline & $1^{\text {ry }}$ or $2^{\text {ry }}$ infection code & $0.630(0.611-0.648)$ & $0.776(0.757-0.795)$ & $0.633(0.612-0.654)$ \\
\hline & No infection code & $0.658(0.642-0.675)$ & $0.803(0.785-0.821)$ & $0.669(0.650-0.689)$ \\
\hline \multirow{5}{*}{ In-hospital Mortality or ICU $\geq 3$ days } & All admissions & $0.644(0.634-0.655)$ & $0.800(0.789-0.810)$ & $0.654(0.642-0.667)$ \\
\hline & $1^{\text {ry }}$ infection code & $0.670(0.649-0.690)$ & $0.830(0.812-0.849)$ & $0.681(0.658-0.704)$ \\
\hline & $2^{\text {ry }}$ infection code & $0.596(0.574-0.618)$ & $0.724(0.701-0.747)$ & $0.608(0.583-0.633)$ \\
\hline & $1^{\text {ry }}$ or $2^{\text {ry }}$ infection code & $0.632(0.617-0.646)$ & $0.784(0.769-0.799)$ & $0.638(0.621-0.655)$ \\
\hline & No infection code & $0.652(0.637-0.668)$ & $0.802(0.786-0.819)$ & $0.663(0.646-0.681)$ \\
\hline \multirow{5}{*}{ Mortality within 24 hours } & All admissions & $0.770(0.749-0.792)$ & $0.898(0.881-0.914)$ & $0.793(0.769-0.816)$ \\
\hline & $1^{\text {ry }}$ infection code & $0.827(0.782-0.871)$ & $0.946(0.916-0.972)$ & $0.865(0.819-0.910)$ \\
\hline & $2^{\text {ry }}$ infection code & $0.753(0.680-0.828)$ & $0.910(0.855-0.955)$ & $0.776(0.694-0.857)$ \\
\hline & $1^{\text {ry }}$ or $2^{\text {ry }}$ infection code & $0.802(0.763-0.840)$ & $0.936(0.909-0.960)$ & $0.833(0.790-0.874)$ \\
\hline & No infection code & $0.752(0.726-0.778)$ & $0.878(0.856-0.900)$ & $0.770(0.742-0.798)$ \\
\hline \multirow{5}{*}{ In-hospital Mortality or unanticipated ICU admission } & All admissions & $0.629(0.620-0.638)$ & $0.780(0.771-0.790)$ & $0.635(0.624-0.645)$ \\
\hline & $1^{\text {ry }}$ infection code & $0.663(0.645-0.681)$ & $0.823(0.806-0.839)$ & $0.673(0.652-0.693)$ \\
\hline & $2^{\text {ry }}$ infection code & $0.590(0.572-0.608)$ & $0.719(0.699-0.739)$ & $0.596(0.576-0.617)$ \\
\hline & $1^{\text {ry }}$ or $2^{\text {ry }}$ infection code & $0.623(0.611-0.636)$ & $0.775(0.762-0.788)$ & $0.626(0.611-0.640)$ \\
\hline & No infection code & $0.628(0.615-0.641)$ & $0.771(0.757-0.784)$ & $0.634(0.620-0.649)$ \\
\hline \multirow{5}{*}{$\begin{array}{r}\text { Mortality within } 24 \text { hours or unanticipated ICU admission } \\
\text { within } 24 \text { hours }\end{array}$} & All admissions & $0.695(0.682-0.708)$ & $0.843(0.832-0.855)$ & $0.711(0.696-0.725)$ \\
\hline & $1^{\text {ry }}$ infection code & $0.742(0.717-0.768)$ & $0.895(0.877-0.913)$ & $0.768(0.741-0.796)$ \\
\hline & $2^{\text {ry }}$ infection code & $0.653(0.623-0.683)$ & $0.793(0.764-0.822)$ & $0.669(0.635-0.702)$ \\
\hline & $1^{\text {ry }}$ or $2^{\text {ry }}$ infection code & $0.702(0.682-0.721)$ & $0.855(0.838-0.871)$ & $0.719(0.698-0.741)$ \\
\hline & No infection code & $0.681(0.663-0.700)$ & $0.822(0.804-0.839)$ & $0.694(0.674-0.714)$ \\
\hline
\end{tabular}




\section{Supplemental Digital Content (in order of its citation in the manuscript)}

Supplementary Table 1: Categorisation of admissions

Supplementary Table 2: Twenty-five most common infection diagnoses in database

Supplementary Table 3: Twenty-five most common non-infection diagnoses in database

Supplementary Figure 1: The distribution of patient age, categorised by no infection code, or primary and secondary infection codes at any point during an admission.

Supplementary Figure 2. The distribution of quick Sequential [Sepsis-related] Organ Failure Assessment (qSOFA) (Figure 2a), National Early Warning Score (NEWS) (Figure 2b) and quick National Early Warning Score (qNEWS) (Figure 2c) values and observed risk of observed risk of in-hospital death categorised by no, primary and secondary infection codes at any point during an admission.

Supplementary Figure 3. The distribution of quick Sequential [Sepsis-related] Organ Failure Assessment (qSOFA) (Figure 3a), National Early Warning Score (NEWS) (Figure 3b) and quick National Early Warning Score (qNEWS) (Figure 3c) values and observed risk of death within 24h of an observation set categorised by no, primary and secondary infection codes at any point during an admission.

Supplementary Figure 4. Plot of qSOFA (Figure 4a), NEWS (Figure 4b) and qNEWS (Figure 4c) values and percentage of admissions with primary infection, secondary infection and no infection.

Supplementary Figure 5. Pictorial display of the area under the receiver operator characteristics curve (AUROC) values for quick Sequential [Sepsis-related] Organ Failure Assessment (qSOFA) score, the National Early Warning Score (NEWS) and the quick National Early Warning Score (qNEWS) for all admissions (Figure 5a), non-elective admission to medicine (Figure 5b) and non-elective admission to surgery (Figure $5 \mathrm{c}$ ) for a range of outcomes.

Supplementary Figure 6. Efficiency curves for quick Sequential [Sepsis-related] Organ Failure Assessment (qSOFA) score and National Early Warning Score (NEWS) for the primary outcome of inhospital death in a) all admissions b) admissions with infection c) admissions without infection. These plot workload (trigger rate) against the sensitivity (both as percentages) of qSOFA and NEWS. Each point on the efficiency curve for each score represents a score value from 0 to 3 (for qSOFA) and 0 to 20 (for NEWS $)$, starting with a value of 0 at the top right. Trigger rate $=($ [true positive + false positive]/[true positive + false positive + true negative + false negative]). The closer the efficiency curve is to the lower right corner, the higher the efficiency of the test (i.e., more outcomes are detected for a lower trigger rate). 
Supplementary Table 4: Area under the receiver operator characteristics curve for quick Sequential [Sepsis-related] Organ Failure Assessment (qSOFA) score, the National Early Warning Score (NEWS) and the quick National Early Warning Score (qNEWS) for a range of outcomes. A comparison of performance between direct admissions and those via the emergency department (ED).

Supplementary Table 5: Area under the receiver operator characteristics curve for quick Sequential [Sepsis-related] Organ Failure Assessment (qSOFA) score, the National Early Warning Score (NEWS) and the quick National Early Warning Score (qNEWS) for a range of outcomes for all admissions, including those where there were no vital signs observations taken in the 24 hours prior to discharge.

Supplementary Table 6: Sensitivity, false positive rates and positive predictive values at each threshold of NEWS/qSOFA for the primary outcome of in-hospital mortality. 


\section{Supplementary Table 1: Categorisation of admissions}

\begin{tabular}{|l|}
\hline Surgical Specialties \\
\hline General Surgery \\
\hline Urology \\
\hline Renal Surgery \& Transplantation \\
\hline Colorectal \\
\hline Upper Gastrointestinal \\
\hline Vascular \\
\hline Trauma \& Orthopaedics \\
\hline \\
\hline Medical Specialties \\
\hline General Medicine \\
\hline Gastroenterology \\
\hline Endocrinology \\
\hline Clinical Haematology \\
\hline Rehabilitation \\
\hline Cardiology \\
\hline Respiratory (Thoracic) Medicine \\
\hline Nephrology \\
\hline Medical Oncology \\
\hline Neurology \\
\hline Rheumatology \\
\hline Geriatric Medicine \\
\hline
\end{tabular}


Supplementary Table 2: Twenty-five most common infection diagnoses in database

\begin{tabular}{|c|c|c|c|c|}
\hline $\begin{array}{l}\text { ICD-10 } \\
\text { Code }\end{array}$ & $\begin{array}{l}\text { Admissions } \\
\text { (n) }\end{array}$ & ICD-10 name & Clinical Classifications Software (CCS) name & $\begin{array}{l}\text { Mortality } \\
(\%)\end{array}$ \\
\hline N39.0 & 7228 & Urinary tract infection, site not specified & Urinary tract infections & 3.40 \\
\hline J18.1 & 6160 & Lobar pneumonia, unspecified & $\begin{array}{l}\text { Pneumonia (except that caused by tuberculosis or sexually } \\
\text { transmitted disease) }\end{array}$ & 14.35 \\
\hline J18.9 & 4453 & Pneumonia, unspecified & $\begin{array}{c}\text { Pneumonia (except that caused by tuberculosis or sexually } \\
\text { transmitted disease) }\end{array}$ & 17.45 \\
\hline J44.0 & 3200 & $\begin{array}{l}\text { Chronic obstructive pulmonary disease with acute lower } \\
\text { respiratory infection }\end{array}$ & Chronic obstructive pulmonary disease and bronchiectasis & 4.25 \\
\hline J22.X & 2941 & Unspecified acute lower respiratory infection & Acute bronchitis & 4.28 \\
\hline L03.1 & 2265 & Cellulitis of other parts of limb & Skin and subcutaneous tissue infections & 2.43 \\
\hline A41.9 & 1949 & Sepsis, unspecified & Septicemia (except in labor) & 15.24 \\
\hline K80.1 & 1214 & Calculus of gallbladder with other cholecystitis & Biliary tract disease & 0.41 \\
\hline T81.4 & 1092 & Infection following a procedure, not elsewhere classified & Complications of surgical procedures or medical care & 0.55 \\
\hline J03.9 & 1043 & Acute tonsillitis, unspecified & Acute and chronic tonsillitis & 0 \\
\hline K80.0 & 869 & Calculus of gallbladder with acute cholecystitis & Biliary tract disease & 0.69 \\
\hline N12.X & 724 & $\begin{array}{l}\text { Tubulo-interstitial nephritis, not specified as acute or } \\
\text { chronic }\end{array}$ & Nephritis; nephrosis; renal sclerosis & 0.28 \\
\hline J69.0 & 675 & Pneumonitis due to food and vomit & Aspiration pneumonitis; food/vomitus & 29.04 \\
\hline J36.X & 659 & Peritonsillar abscess & Acute and chronic tonsillitis & 0 \\
\hline K35.8 & 636 & Acute appendicitis, other and unspecified & Appendicitis and other appendiceal conditions & 0 \\
\hline L02.4 & 519 & Cutaneous abscess, furuncle and carbuncle of limb & Skin and subcutaneous tissue infections & 0.19 \\
\hline K61.0 & 515 & Anal abscess & Anal and rectal conditions & 0 \\
\hline K37.X & 466 & Unspecified appendicitis & Appendicitis and other appendiceal conditions & 0.21 \\
\hline L02.2 & 432 & Cutaneous abscess, furuncle and carbuncle of trunk & Skin and subcutaneous tissue infections & 1.16 \\
\hline L05.0 & 385 & Pilonidal cyst with abscess & Skin and subcutaneous tissue infections & 0 \\
\hline J18.0 & 363 & Bronchopneumonia, unspecified & $\begin{array}{c}\text { Pneumonia (except that caused by tuberculosis or sexually } \\
\text { transmitted disease) }\end{array}$ & 64.74 \\
\hline K35.3 & 355 & Acute appendicitis with localized peritonitis & Appendicitis and other appendiceal conditions & 0.28 \\
\hline A04.7 & 345 & Enterocolitis due to Clostridium difficile & Intestinal infection & 5.80 \\
\hline K81.0 & 337 & Acute cholecystitis & Biliary tract disease & 2.67 \\
\hline K35.9 & 319 & Acute appendicitis, unspecified & Appendicitis and other appendiceal conditions & 0 \\
\hline
\end{tabular}

ICD-10 codes were subsequently mapped to Clinical Coding Software (CCS) groups using tables ${ }^{\mathrm{a}}$ available from NHS Digital ${ }^{\mathrm{b}}$

${ }^{a}$ Clinical Coding Software (CCS) groups (http://hcup-us.ahrq.gov/toolssoftware/icd 10/ccs icd 10.jsp).

bSummary Hospital-Level Indicator, NHS Digital (http://content.digital.nhs.uk/SHMI) 
Supplementary Table 3: Twenty-five most common non-infection diagnoses in database

\begin{tabular}{|c|c|c|c|c|}
\hline $\begin{array}{l}\text { ICD-10 } \\
\text { Code }\end{array}$ & $\begin{array}{l}\text { Admissions } \\
\text { (n) }\end{array}$ & ICD-10 name & Clinical Classifications Software (CCS) name & $\begin{array}{l}\text { Mortality } \\
(\%)\end{array}$ \\
\hline 148.X & 4697 & Atrial fibrillation and flutter & Cardiac dysrhythmias & 4.75 \\
\hline R07.4 & 4428 & Chest pain, unspecified & Nonspecific chest pain & 0.90 \\
\hline R55.X & 4171 & Syncope and collapse & Syncope & 3.33 \\
\hline M17.9 & 3954 & Gonarthrosis, unspecified & Osteoarthritis & 0.51 \\
\hline Z86.4 & 3792 & Personal history of psychoactive substance abuse & Residual codes; unclassified & 5.88 \\
\hline 163.9 & 3684 & Cerebral infarction, unspecified & Acute cerebrovascular disease & 5.05 \\
\hline R10.4 & 3543 & Other and unspecified abdominal pain & Abdominal pain & 1.47 \\
\hline R29.6 & 3391 & Tendency to fall, not elsewhere classified & Other connective tissue disease & 6.08 \\
\hline I10.X & 3294 & Essential (primary) hypertension & Essential hypertension & 9.72 \\
\hline 121.9 & 3278 & Acute myocardial infarction, unspecified & Acute myocardial infarction & 5.74 \\
\hline M16.9 & 3223 & Coxarthrosis, unspecified & Osteoarthritis & 0.40 \\
\hline S72.00 & 3097 & Fracture of neck of femur & Fracture of neck of femur (hip) & 3.97 \\
\hline I25.1 & 3090 & Atherosclerotic heart disease & Coronary atherosclerosis and other heart disease & 1.33 \\
\hline $\mathbf{1 5 0 . 0}$ & 2677 & Congestive heart failure & Congestive heart failure; nonhypertensive & 12.44 \\
\hline Z86.7 & 2675 & Personal history of diseases of the circulatory system & Residual codes; unclassified & 5.50 \\
\hline J44.1 & 2534 & $\begin{array}{l}\text { Chronic obstructive pulmonary disease with acute } \\
\text { exacerbation, unspecified }\end{array}$ & Chronic obstructive pulmonary disease and bronchiectasis & 3.83 \\
\hline N17.9 & 2473 & Acute renal failure, unspecified & Acute and unspecified renal failure & 12.74 \\
\hline J45.9 & 2261 & Asthma, unspecified & Asthma & 2.43 \\
\hline K59.0 & 2130 & Constipation & Other gastrointestinal disorders & 2.3 \\
\hline K92.2 & 2112 & Gastrointestinal haemorrhage, unspecified & Gastrointestinal hemorrhage & 5.87 \\
\hline R07.3 & 2073 & Other chest pain & Nonspecific chest pain & 0.29 \\
\hline R10.3 & 2064 & Pain localized to other parts of lower abdomen & Abdominal pain & 0.34 \\
\hline C50.9 & 1997 & Malignant neoplasm: Breast, unspecified & Cancer of breast & 1.80 \\
\hline R06.0 & 1881 & Dyspnoea & Other upper respiratory disease & 5.90 \\
\hline Z92.1 & 1840 & $\begin{array}{l}\text { Personal history of long-term (current) use of } \\
\text { anticoagulants }\end{array}$ & Residual codes; unclassified & 4.84 \\
\hline
\end{tabular}

ICD-10 codes were subsequently mapped to Clinical Coding Software (CCS) groups using tables ${ }^{\mathrm{a}}$ available from NHS Digital $^{\mathrm{b}}$

${ }^{a}$ Clinical Coding Software (CCS) groups (http://hcup-us.ahrq.gov/toolssoftware/icd 10/ccs icd 10.jsp).

bSummary Hospital-Level Indicator, NHS Digital (http://content.digital.nhs.uk/SHMI) 
Supplementary Figure 1: The distribution of patient age, categorised by no infection code, or primary and secondary infection codes at any point during an admission.

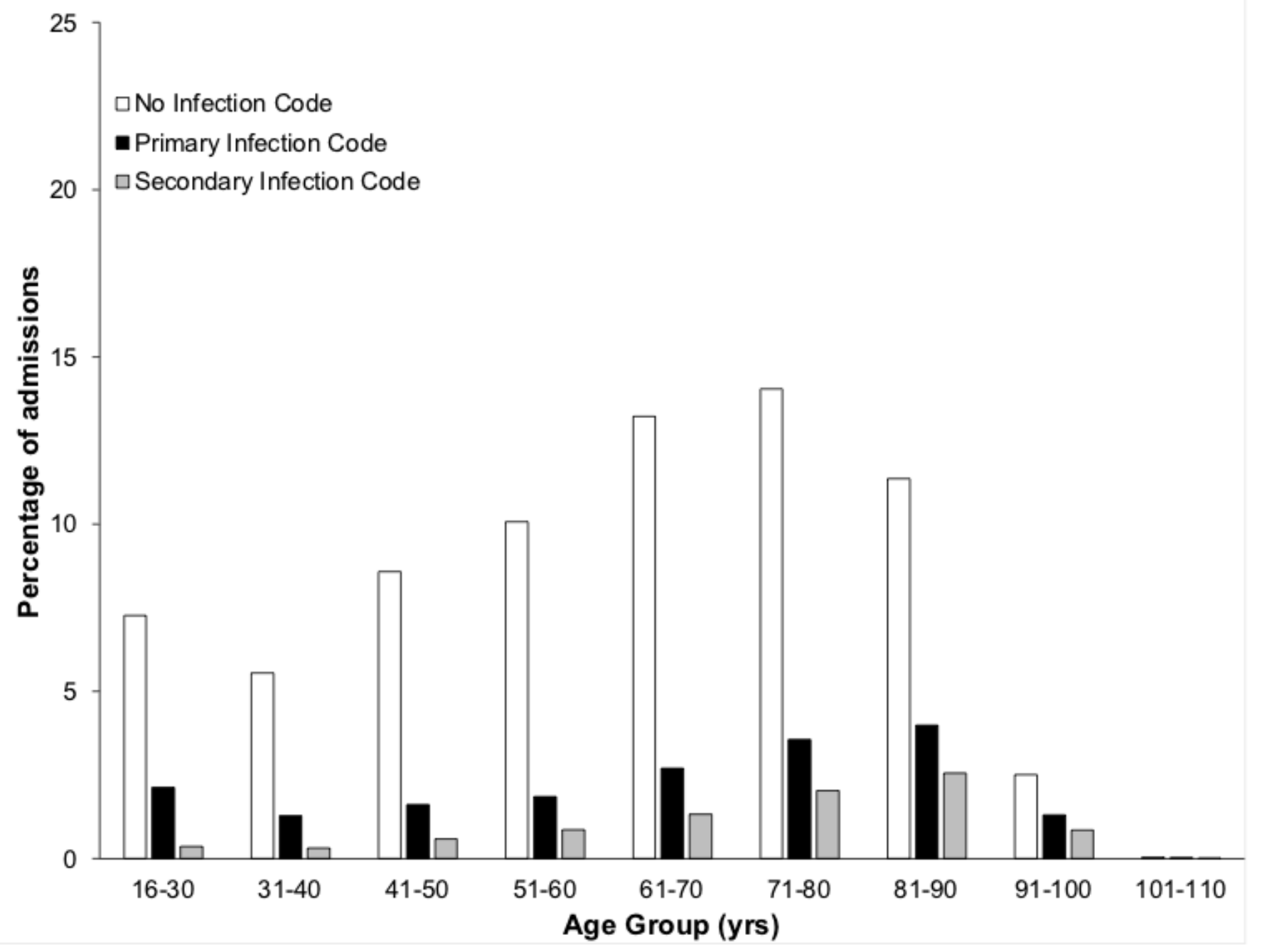


Supplementary Figure 2a. The distribution of quick Sequential [Sepsis-related] Organ Failure Assessment (qSOFA) values and observed risk of observed risk of in-hospital death categorised by no, primary and secondary infection codes at any point during an admission.

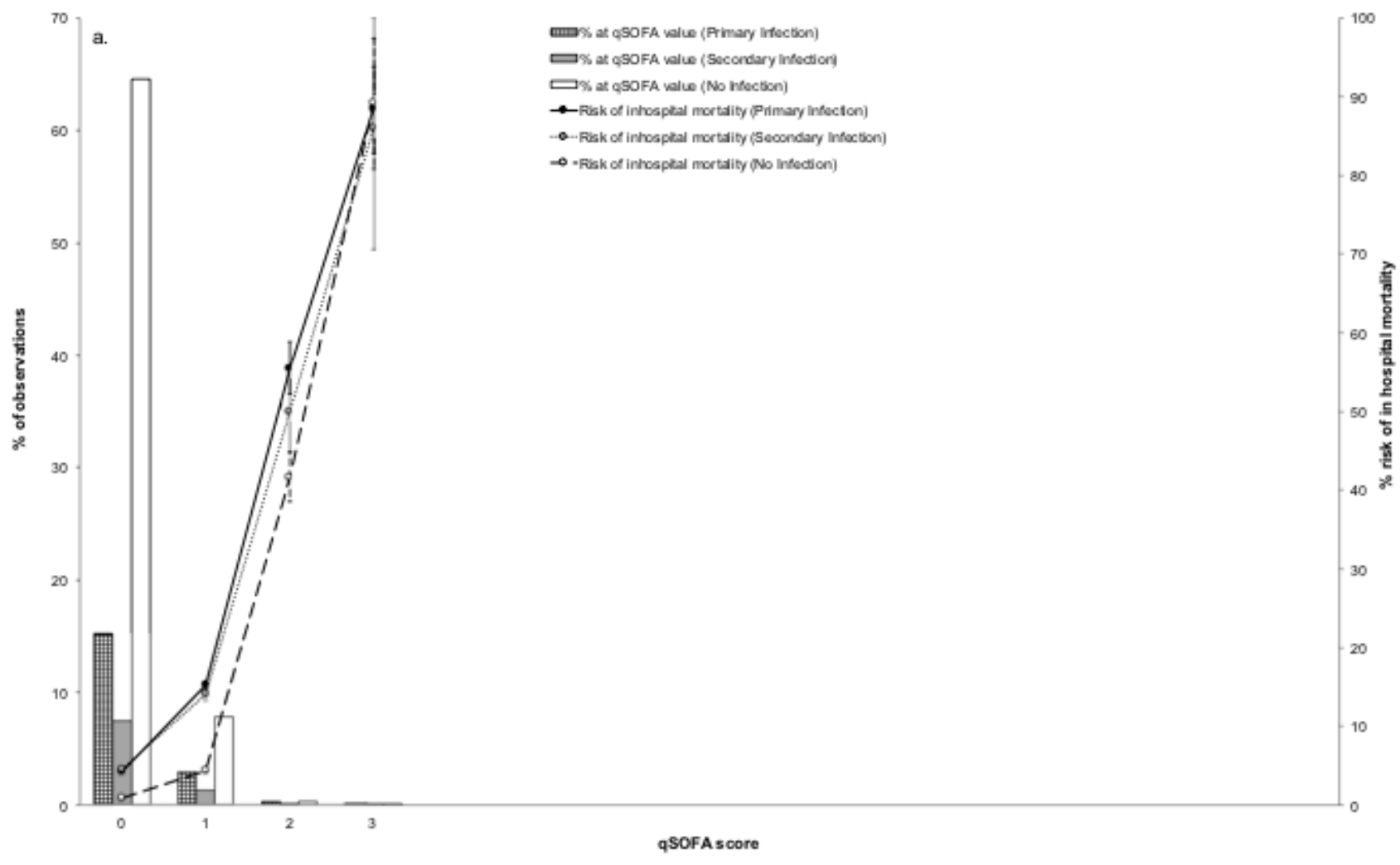


Supplementary Figure 2b. The distribution of National Early Warning Score (NEWS) (values and observed risk of observed risk of inhospital death categorised by no, primary and secondary infection codes at any point during an admission.

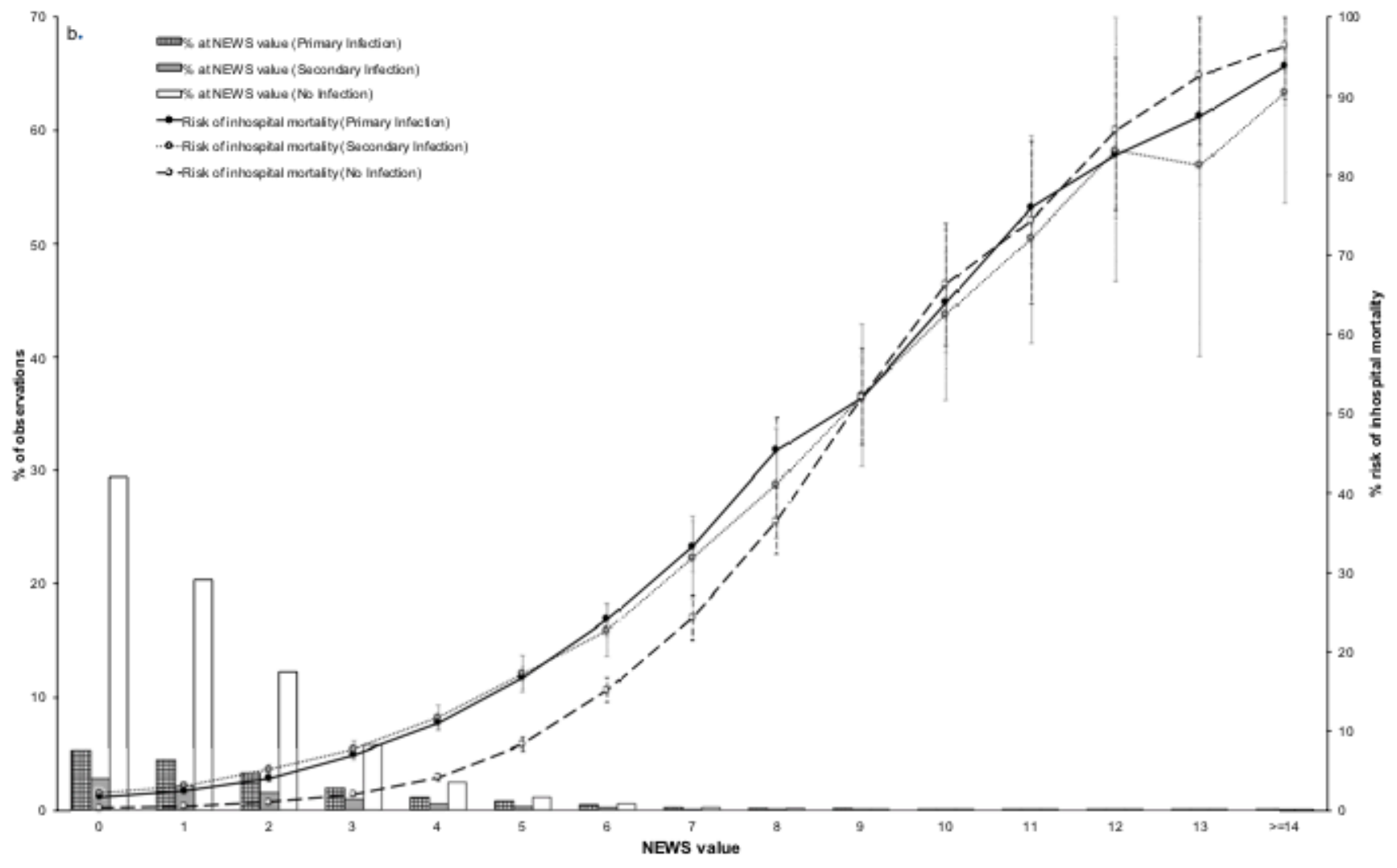


Supplementary Figure 2c. The distribution of quick National Early Warning Score (qNEWS) values and observed risk of observed risk of in-hospital death categorised by no, primary and secondary infection codes at any point during an admission.

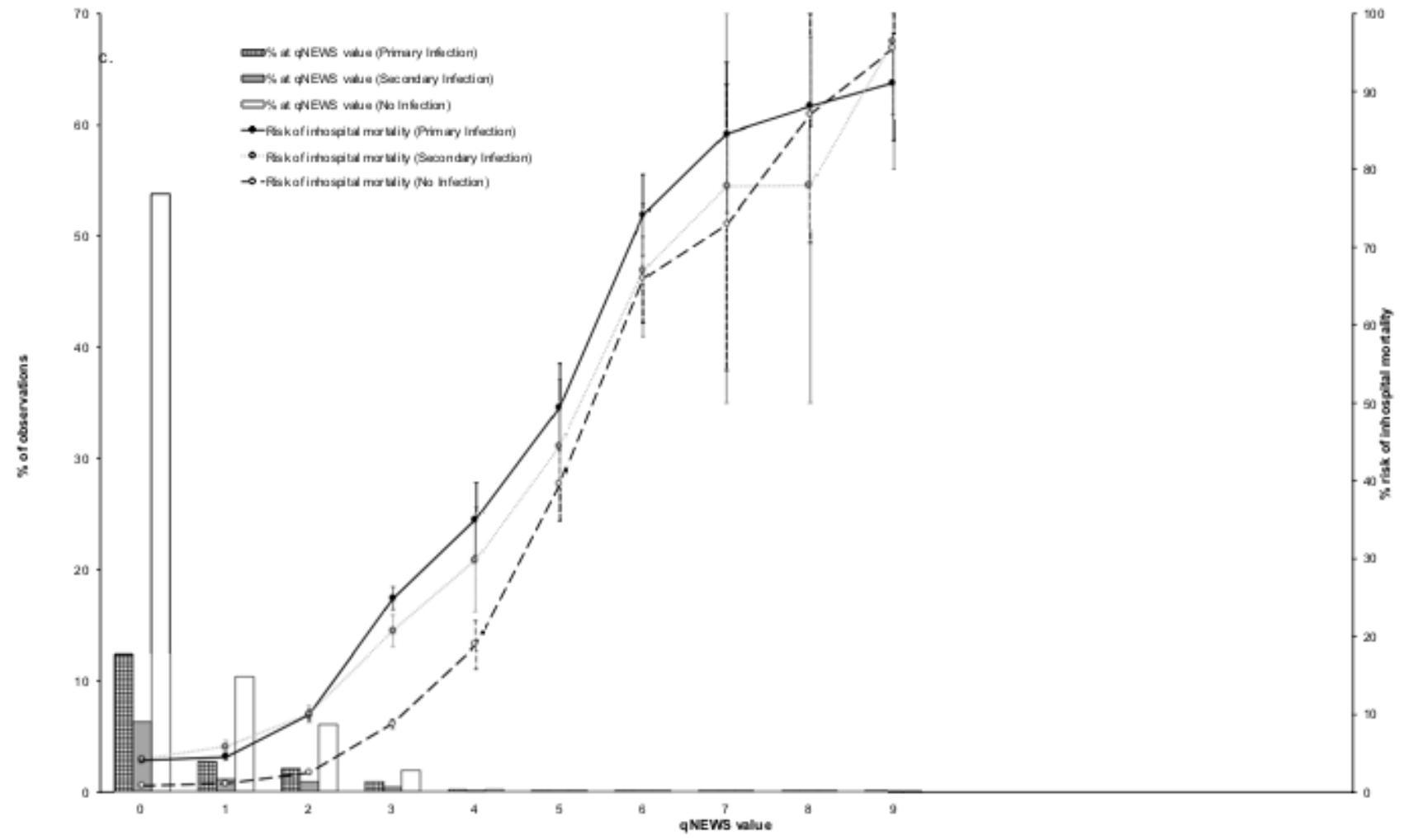


Supplementary Figure 3a. The distribution of quick Sequential [Sepsis-related] Organ Failure Assessment (qSOFA) values and observed risk of death within $24 \mathrm{~h}$ of an observation set categorised by no, primary and secondary infection codes at any point during an admission.

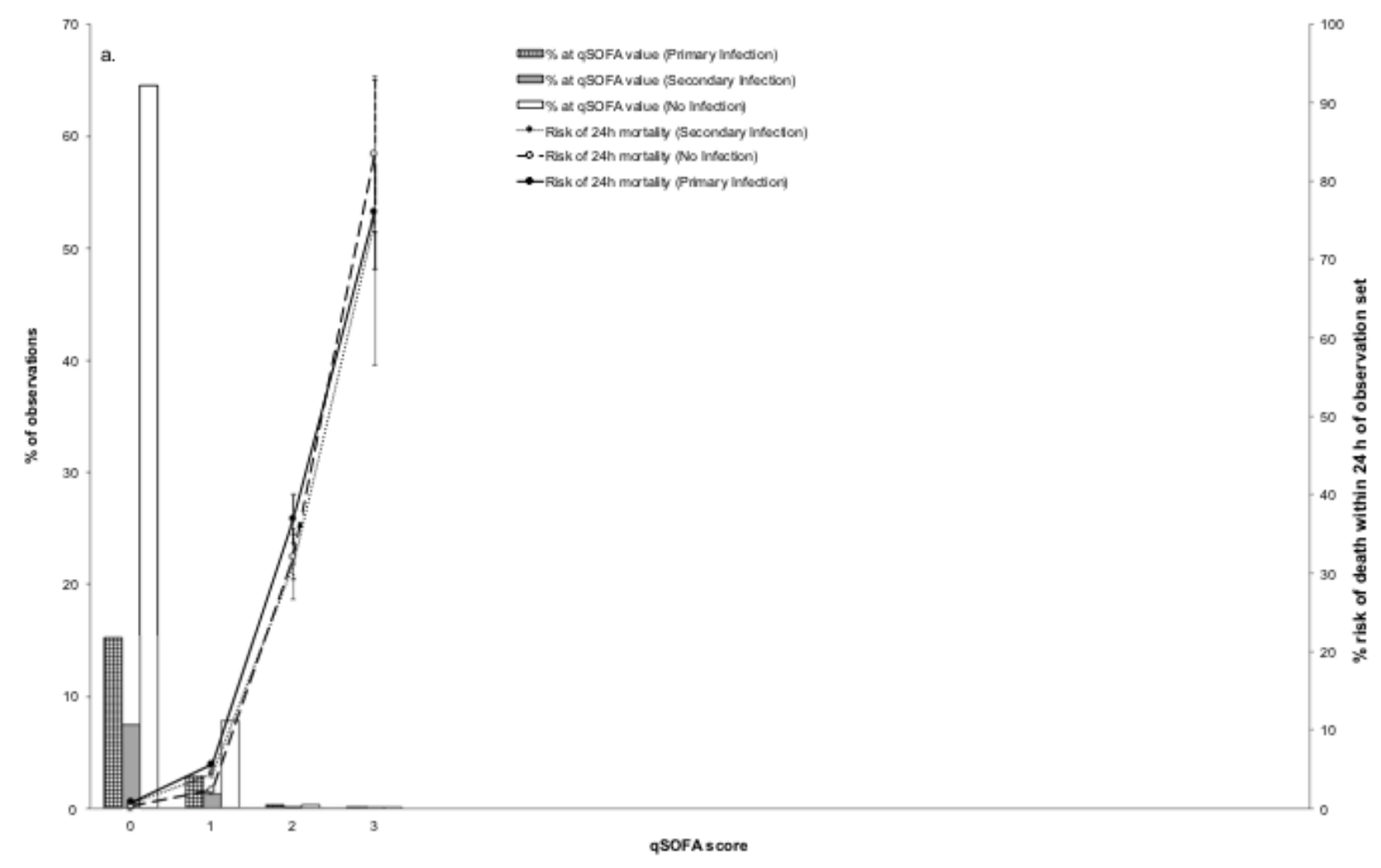


Supplementary Figure 3b. The distribution of National Early Warning Score (NEWS) values and observed risk of death within 24h of an observation set categorised by no, primary and secondary infection codes at any point during an admission.

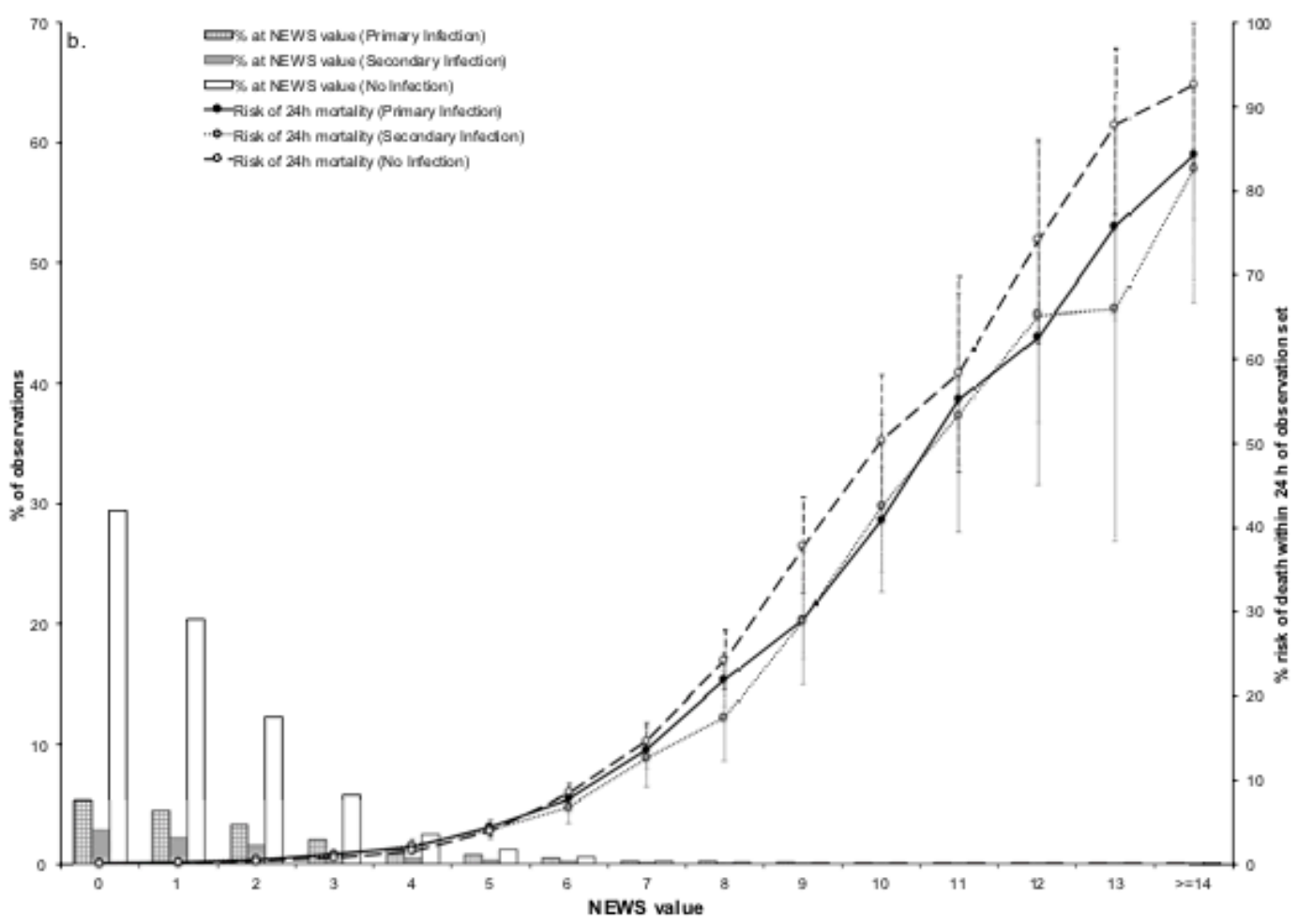


Supplementary Figure 3c. The distribution of quick National Early Warning Score (qNEWS) values and observed risk of death within $24 \mathrm{~h}$ of an observation set categorised by no, primary and secondary infection codes at any point during an admission.

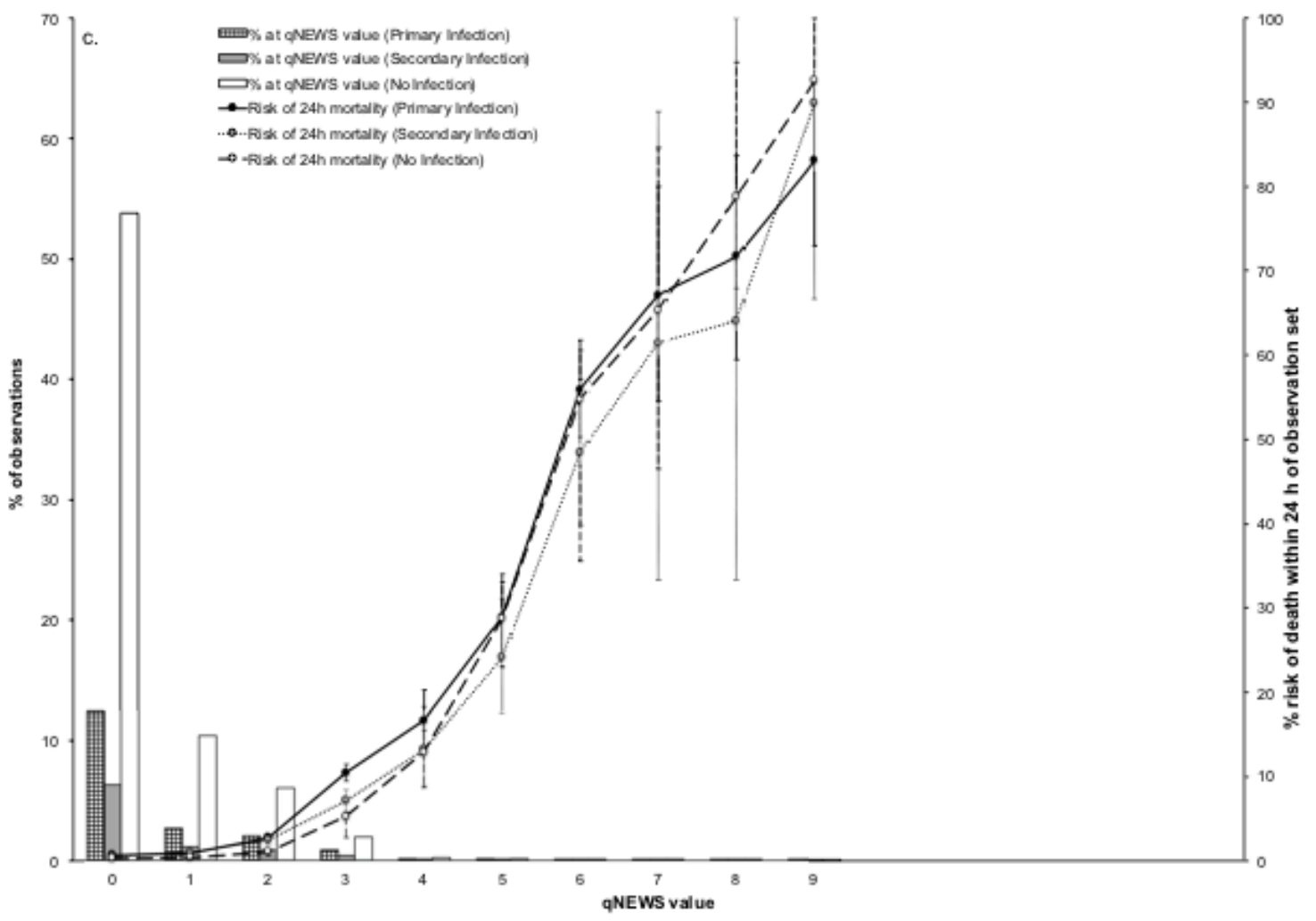


Supplementary Figure 4a. Plot of quick Sequential [Sepsis-related] Organ Failure Assessment (qSOFA) values and percentage of admissions with primary infection, secondary infection and no infection.

a.

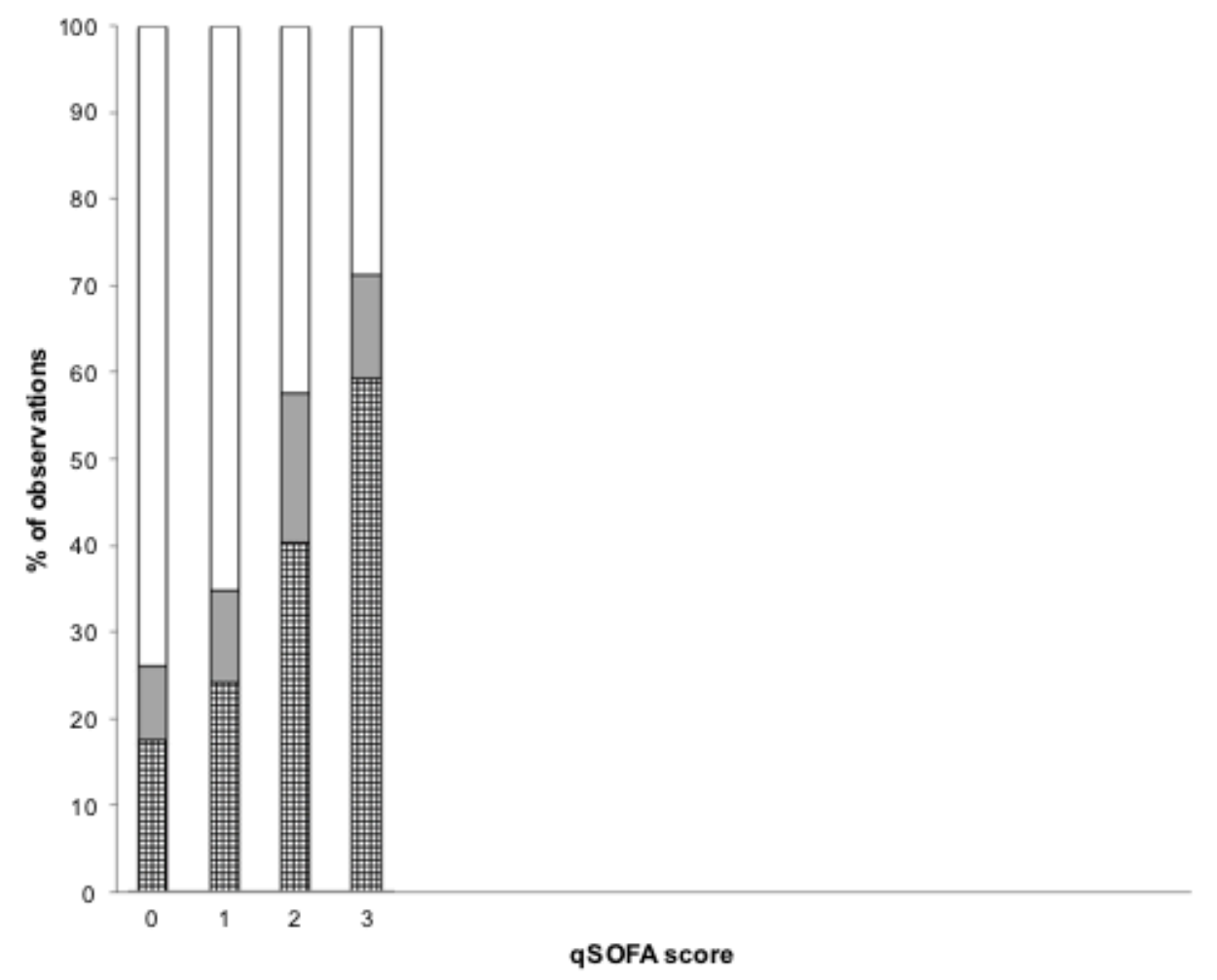

口No infection

esecondary infection

Primary infection 
Supplementary Figure 4b. Plot of National Early Warning Score (NEWS) values and percentage of admissions with primary infection, secondary infection and no infection.

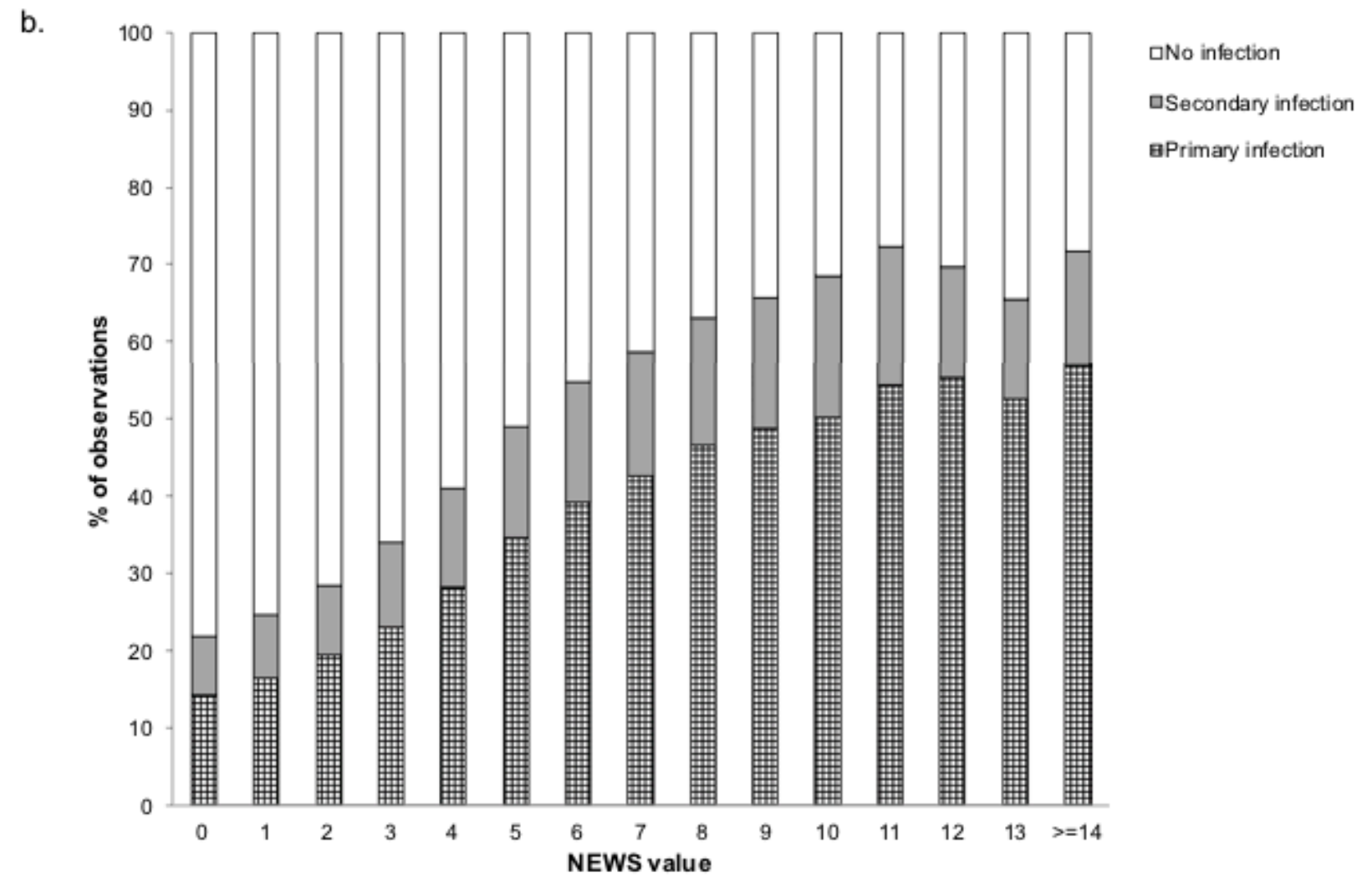


Supplementary Figure 4c. Plot of quick National Early Warning Score (qNEWS) values and percentage of admissions with primary infection, secondary infection and no infection.

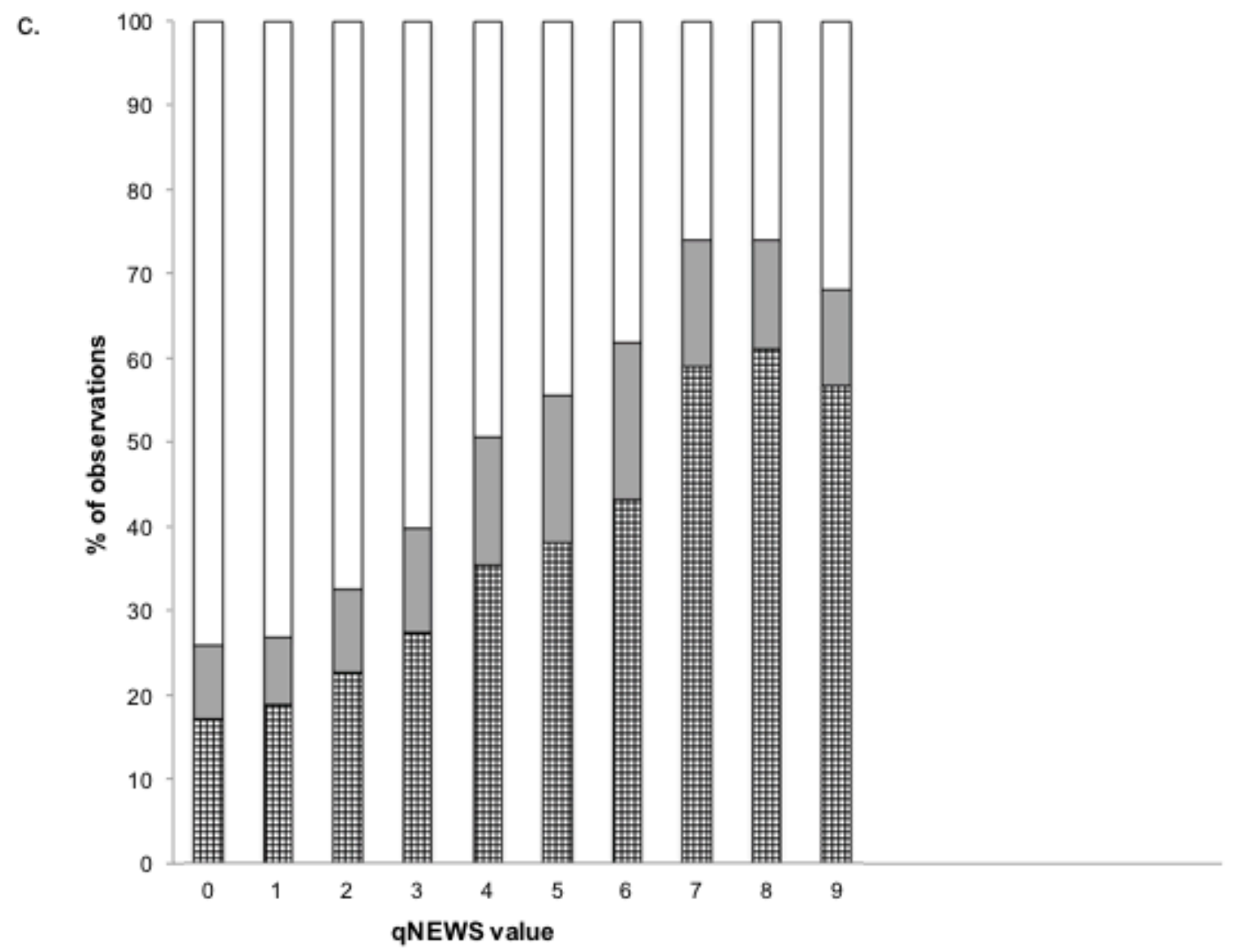

םNo infection

usecondary infection

هPrimary infection 
Supplementary Figure 5a. Pictorial display of area under the receiver operator characteristics curve (AUROC) values for quick Sequential [Sepsis-related] Organ Failure Assessment (qSOFA) score (Figure 5a), the National Early Warning Score (NEWS) (Figure 5b) and the quick National Early Warning Score (qNEWS) (Figure 5c) for a range of outcomes for the all admissions group.

All admissions

In-hospital Mortality

All admissions

1ry infection code

2 ry infection code

1 ry or 2 ry infection

No infection code

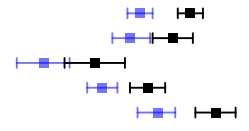

In-hospital Mortality or ICU $\geq 3$ days

All admissions

1 ry infection code

2 ry infection code

1 ry or 2 ry infection code

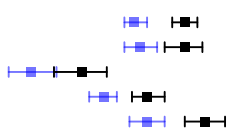

In-hospital Mortality or unanticipated ICU admission

All admissions

1 ry infection code

2 ry infection code

1 ry or 2 ry infection code

No infection code

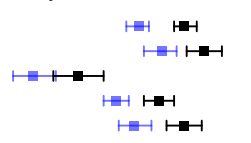

Mortality within 24 hours

All admissions

1 ry infection code

2 ry infection code

1 ry or 2ry infection code

No infection code

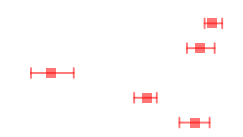

Mortality within 24 hours or unanticipated ICU admission

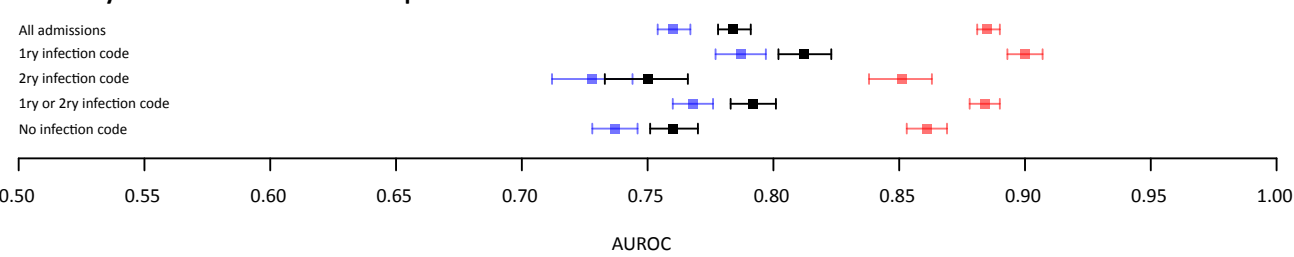

NEWS $\square$ qSOFA

qNEWS 
Supplementary Figure 5b. Pictorial display of area under the receiver operator characteristics curve (AUROC) values for quick Sequential [Sepsis-related] Organ Failure Assessment (qSOFA) score (Figure 5a), the National Early Warning Score (NEWS) (Figure 5b) and the quick National Early Warning Score (qNEWS) (Figure 5c) for a range of outcomes for the non-elective admissions to medicine group.

In-hospital Mortality

All admissions

1 ry infection code

2 ry infection code

1 ry or 2 ry infection code

No infection code

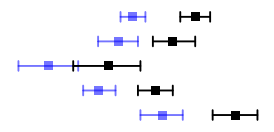

In-hospital Mortality or ICU $\geq 3$ days

All admissions

1 ry infection code

2 ry infection code

1 ry or 2 ry infection code

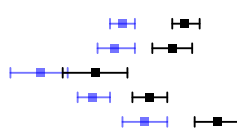

In-hospital Mortality or unanticipated ICU admission

All admissions

1 ry infection code

$2 r y$ infection code

1 ry or 2 ry infection cod

$\mapsto-1 \mapsto-1$

No infection code

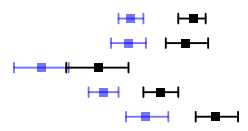

Mortality within 24 hours

All admissions

1 ry infection code

2 ry infection code

1 ry or 2ry infection code

No infection code

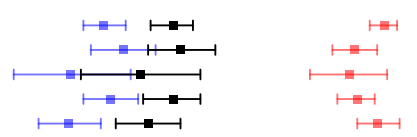

Mortality within 24 hours or unanticipated ICU admission

All admissions

1 ry infection code

2 ry infection code

1 ry or 2 ry infection code

No infection code

\begin{tabular}{|c|c|c|c|c|c|c|c|c|c|c|}
\hline 1 & $T$ & $T$ & $T$ & $T$ & $T$ & $T$ & $T$ & $T$ & $T$ & \\
\hline 50 & 0.55 & 0.60 & 0.65 & 0.70 & 0.75 & 0.80 & 0.85 & 0.90 & 0.95 & 1.0 \\
\hline
\end{tabular}

AUROC 
Supplementary Figure 5c. Pictorial display of area under the receiver operator characteristics curve (AUROC) values for quick Sequential [Sepsis-related] Organ Failure Assessment (qSOFA) score (Figure 5a), the National Early Warning Score (NEWS) (Figure 5b) and the quick National Early Warning Score (qNEWS) (Figure 5c) for a range of outcomes for the for the non-elective admissions to surgery group.

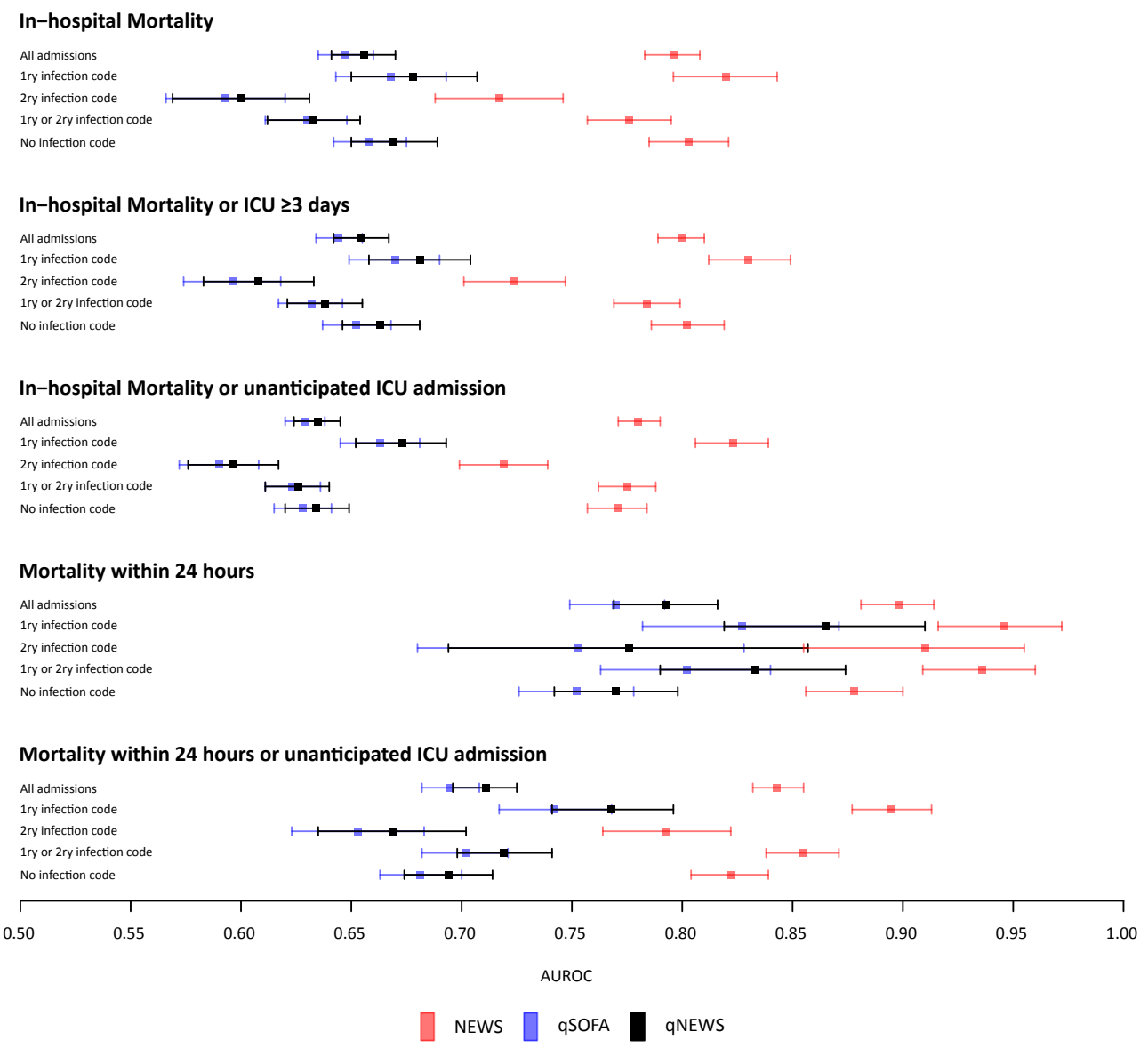


Supplementary Figure 6. Efficiency curves for quick Sequential [Sepsis-related] Organ Failure Assessment (qSOFA) score and National Early Warning Score (NEWS) for the primary outcome of in-hospital death in a) all admissions b) admissions with infection c) admissions without infection. These plot workload (trigger rate) against the sensitivity (both as percentages) of qSOFA and NEWS. Each point on the efficiency curve for each score represents a score value from 0 to 3 (for qSOFA) and 0 to 20 (for NEWS), starting with a value of 0 at the top right. Trigger rate $=$ ([true positive + false positive]/[true positive + false positive + true negative + false negative]). The closer the efficiency curve is to the lower right corner, the higher the efficiency of the test (i.e., more outcomes are detected for a lower trigger rate).
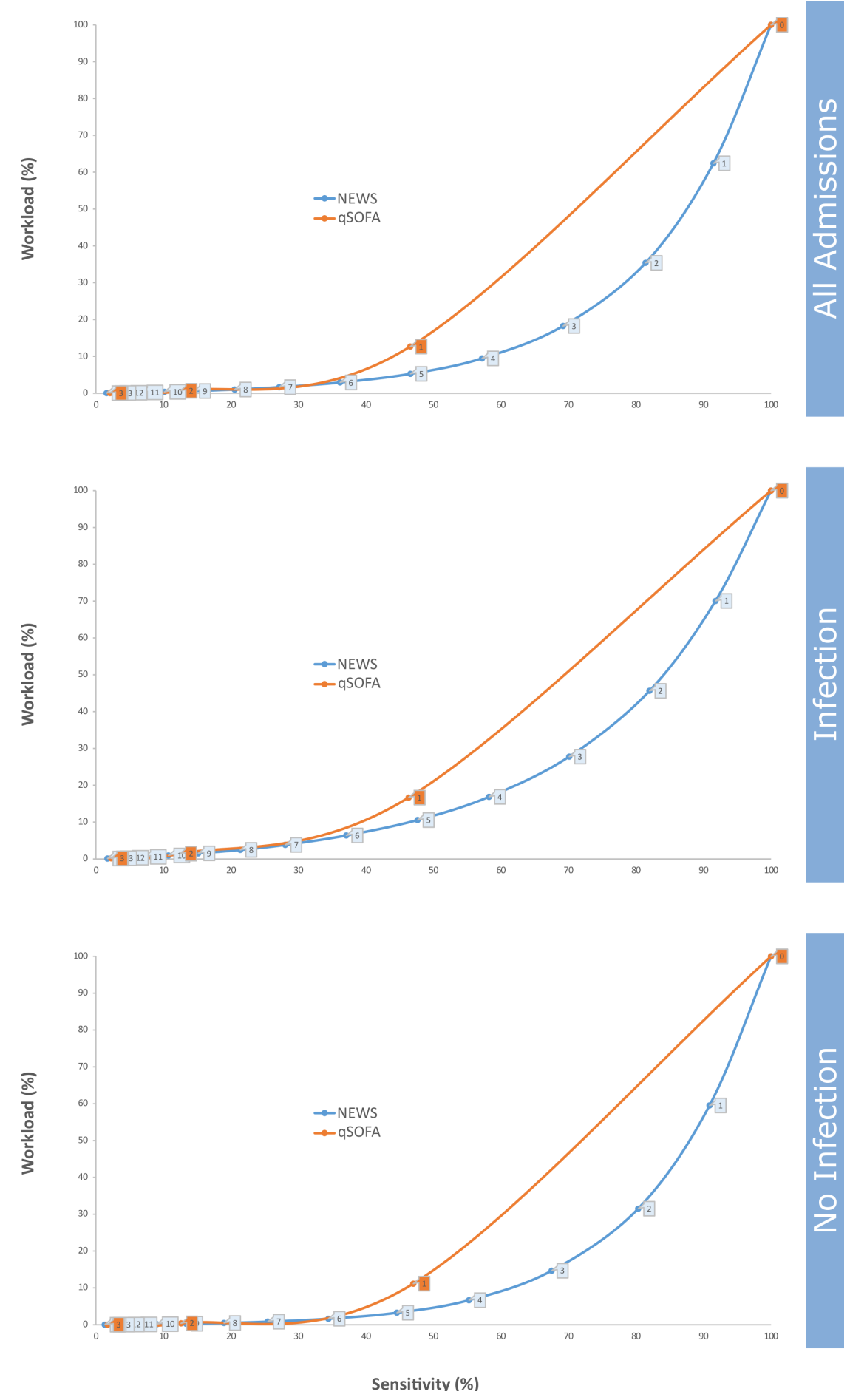
Supplementary Table 4: Area under the receiver operator characteristics curve for quick Sequential [Sepsis-related] Organ Failure Assessment (qSOFA) score, the National Early Warning Score (NEWS) and the quick National Early Warning Score (qNEWS) for a range of outcomes. A comparison of performance between direct admissions and those via the emergency department (ED).

\begin{tabular}{|c|c|c|c|c|}
\hline All admissions & & qSOFA & NEWS & qNEWS \\
\hline Outcome & & & & \\
\hline \multirow{5}{*}{ In-hospital Mortality } & All admissions & \begin{tabular}{|l|}
0.681 \\
$(0.676-$ \\
$0.686)$ \\
\end{tabular} & $\begin{array}{l}0.825 \\
(0.821- \\
0.829) \\
\end{array}$ & $\begin{array}{l}0.701 \\
(0.696- \\
0.706) \\
\end{array}$ \\
\hline & $\begin{array}{l}1^{\text {ry }} \text { infection } \\
\text { code }\end{array}$ & \begin{tabular}{|l|}
0.677 \\
$(0.670-$ \\
$0.685)$
\end{tabular} & $\begin{array}{l}0.805 \\
(0.799- \\
0.812)\end{array}$ & $\begin{array}{l}0.694 \\
(0.686- \\
0.702)\end{array}$ \\
\hline & $\begin{array}{l}2^{\text {ry }} \text { infection } \\
\text { code }\end{array}$ & \begin{tabular}{|l|}
0.643 \\
$(0.632-$ \\
$0.653)$ \\
\end{tabular} & $\begin{array}{l}0.759 \\
(0.748- \\
0.769)\end{array}$ & $\begin{array}{l}0.663 \\
(0.651- \\
0.675)\end{array}$ \\
\hline & $\begin{array}{l}1^{\text {ry }} \text { or } 2^{\text {ry }} \\
\text { infection code }\end{array}$ & \begin{tabular}{|l|}
0.666 \\
$(0.660-$ \\
$0.672)$ \\
\end{tabular} & $\begin{array}{l}0.790 \\
(0.785- \\
0.796) \\
\end{array}$ & $\begin{array}{l}0.684 \\
(0.677- \\
0.691) \\
\end{array}$ \\
\hline & $\begin{array}{l}\text { No infection } \\
\text { code }\end{array}$ & \begin{tabular}{|l|}
0.688 \\
$(0.680-$ \\
$0.695)$ \\
\end{tabular} & $\begin{array}{l}0.831 \\
(0.825- \\
0.838) \\
\end{array}$ & $\begin{array}{l}0.711 \\
(0.703- \\
0.719) \\
\end{array}$ \\
\hline \multirow{5}{*}{$\begin{array}{r}\text { In-hospital Mortality or ICU } \geq \\
3 \text { days }\end{array}$} & All admissions & \begin{tabular}{|l|}
0.678 \\
$(0.674-$ \\
$0.683)$ \\
\end{tabular} & $\begin{array}{l}0.824 \\
(0.820- \\
0.828) \\
\end{array}$ & $\begin{array}{l}0.698 \\
(0.693- \\
0.703) \\
\end{array}$ \\
\hline & $\begin{array}{l}1^{\text {ry }} \text { infection } \\
\text { code }\end{array}$ & \begin{tabular}{|l|}
0.680 \\
$(0.674-$ \\
$0.687)$ \\
\end{tabular} & $\begin{array}{l}0.810 \\
(0.804- \\
0.816) \\
\end{array}$ & $\begin{array}{l}0.698 \\
(0.690- \\
0.705) \\
\end{array}$ \\
\hline & $\begin{array}{l}2^{\text {ry }} \text { infection } \\
\text { code }\end{array}$ & \begin{tabular}{|l|}
0.637 \\
$(0.628-$ \\
$0.647)$ \\
\end{tabular} & $\begin{array}{l}0.758 \\
(0.749- \\
0.767) \\
\end{array}$ & $\begin{array}{l}0.657 \\
(0.646- \\
0.667)\end{array}$ \\
\hline & $\begin{array}{l}1^{\text {ry }} \text { or } 2^{\text {ry }} \\
\text { infection code }\end{array}$ & \begin{tabular}{|l|}
0.666 \\
$(0.660-$ \\
$0.671)$ \\
\end{tabular} & $\begin{array}{l}0.792 \\
(0.787- \\
0.797) \\
\end{array}$ & $\begin{array}{l}0.683 \\
(0.677- \\
0.690) \\
\end{array}$ \\
\hline & $\begin{array}{l}\text { No infection } \\
\text { code }\end{array}$ & \begin{tabular}{|l|}
0.683 \\
$(0.676-$ \\
$0.690)$ \\
\end{tabular} & $\begin{array}{l}0.826 \\
(0.820- \\
0.833) \\
\end{array}$ & $\begin{array}{l}0.706 \\
(0.698- \\
0.714) \\
\end{array}$ \\
\hline \multirow{5}{*}{ Mortality within 24 hours } & All admissions & \begin{tabular}{|l|}
0.798 \\
$(0.790-$ \\
$0.805)$ \\
\end{tabular} & $\begin{array}{l}0.918 \\
(0.913- \\
0.923) \\
\end{array}$ & $\begin{array}{l}0.823 \\
(0.815- \\
0.831) \\
\end{array}$ \\
\hline & $\begin{array}{l}1^{\text {ry }} \text { infection } \\
\text { code }\end{array}$ & \begin{tabular}{|l|}
0.811 \\
$(0.798-$ \\
$0.823)$ \\
\end{tabular} & $\begin{array}{l}0.919 \\
(0.911- \\
0.926) \\
\end{array}$ & $\begin{array}{l}0.834 \\
(0.821- \\
0.847) \\
\end{array}$ \\
\hline & $\begin{array}{l}2^{\text {ry }} \text { infection } \\
\text { code }\end{array}$ & \begin{tabular}{|l|}
0.789 \\
$(0.768-$ \\
$0.811)$ \\
\end{tabular} & $\begin{array}{l}0.906 \\
(0.891- \\
0.920)\end{array}$ & $\begin{array}{l}0.815 \\
(0.794- \\
0.837) \\
\end{array}$ \\
\hline & $\begin{array}{l}1^{\text {ry }} \text { or } 2^{\text {ry }} \\
\text { infection code }\end{array}$ & \begin{tabular}{|l|}
0.805 \\
$(0.795-$ \\
$0.816)$
\end{tabular} & $\begin{array}{l}0.916 \\
(0.909- \\
0.922)\end{array}$ & $\begin{array}{l}0.829 \\
(0.819- \\
0.841) \\
\end{array}$ \\
\hline & $\begin{array}{l}\text { No infection } \\
\text { code }\end{array}$ & \begin{tabular}{|l|}
0.778 \\
$(0.767-$ \\
$0.789)$ \\
\end{tabular} & $\begin{array}{l}0.904 \\
(0.896- \\
0.912) \\
\end{array}$ & $\begin{array}{l}0.805 \\
(0.794- \\
0.816) \\
\end{array}$ \\
\hline
\end{tabular}




\begin{tabular}{|c|c|c|c|c|}
\hline \multirow{5}{*}{$\begin{array}{r}\text { In-hospital Mortality or } \\
\text { unanticipated ICU admission }\end{array}$} & All admissions & \begin{tabular}{|l|}
0.671 \\
$(0.666-$ \\
$0.675)$ \\
\end{tabular} & $\begin{array}{l}0.813 \\
(0.810- \\
0.817)\end{array}$ & $\begin{array}{l}0.689 \\
(0.685- \\
0.694)\end{array}$ \\
\hline & $\begin{array}{l}1^{\text {ry }} \text { infection } \\
\text { code }\end{array}$ & \begin{tabular}{|l|}
0.680 \\
$(0.673-$ \\
$0.686)$ \\
\end{tabular} & $\begin{array}{l}0.808 \\
(0.803- \\
0.814)\end{array}$ & $\begin{array}{l}0.697 \\
(0.690- \\
0.704)\end{array}$ \\
\hline & $\begin{array}{l}2^{\text {ry }} \text { infection } \\
\text { code }\end{array}$ & $\begin{array}{l}0.629 \\
(0.621- \\
0.638)\end{array}$ & $\begin{array}{l}0.749 \\
(0.741- \\
0.758)\end{array}$ & $\begin{array}{l}0.647 \\
(0.637- \\
0.657)\end{array}$ \\
\hline & $\begin{array}{l}1^{\text {ry }} \text { or } 2^{\text {ry }} \\
\text { infection code }\end{array}$ & \begin{tabular}{|l|}
0.662 \\
$(0.657-$ \\
$0.667)$ \\
\end{tabular} & $\begin{array}{l}0.787 \\
(0.782- \\
0.791)\end{array}$ & $\begin{array}{l}0.679 \\
(0.673- \\
0.685)\end{array}$ \\
\hline & $\begin{array}{l}\text { No infection } \\
\text { code }\end{array}$ & \begin{tabular}{|l|}
0.669 \\
$(0.663-$ \\
$0.676)$ \\
\end{tabular} & $\begin{array}{l}0.806 \\
(0.800- \\
0.812) \\
\end{array}$ & $\begin{array}{l}0.689 \\
(0.682- \\
0.696)\end{array}$ \\
\hline \multirow{5}{*}{$\begin{array}{r}\text { Mortality within } 24 \text { hours or } \\
\text { unanticipated ICU admission } \\
\text { within } 24 \text { hours }\end{array}$} & All admissions & $\begin{array}{l}0.760 \\
(0.754- \\
0.767) \\
\end{array}$ & $\begin{array}{l}0.885 \\
(0.881- \\
0.890)\end{array}$ & $\begin{array}{l}0.784 \\
(0.778- \\
0.791)\end{array}$ \\
\hline & $\begin{array}{l}1^{\text {ry }} \text { infection } \\
\text { code }\end{array}$ & $\begin{array}{l}0.787 \\
(0.777- \\
0.797) \\
\end{array}$ & $\begin{array}{l}0.900 \\
(0.893- \\
0.907)\end{array}$ & $\begin{array}{l}0.812 \\
(0.802- \\
0.823)\end{array}$ \\
\hline & $\begin{array}{l}2^{\text {ry }} \text { infection } \\
\text { code }\end{array}$ & \begin{tabular}{|l|}
0.728 \\
$(0.712-$ \\
$0.744)$ \\
\end{tabular} & $\begin{array}{l}0.851 \\
(0.838- \\
0.863)\end{array}$ & $\begin{array}{l}0.750 \\
(0.733- \\
0.766) \\
\end{array}$ \\
\hline & $\begin{array}{l}1^{\text {ry }} \text { or } 2^{\text {ry }} \\
\text { infection code }\end{array}$ & \begin{tabular}{|l|}
0.768 \\
$(0.760-$ \\
$0.776)$ \\
\end{tabular} & $\begin{array}{l}0.884 \\
(0.878- \\
0.890)\end{array}$ & $\begin{array}{l}0.792 \\
(0.783- \\
0.801)\end{array}$ \\
\hline & $\begin{array}{l}\text { No infection } \\
\text { code }\end{array}$ & \begin{tabular}{|l|}
0.737 \\
$(0.728-$ \\
$0.746)$ \\
\end{tabular} & $\begin{array}{l}0.861 \\
(0.853- \\
0.869)\end{array}$ & $\begin{array}{l}0.760 \\
(0.751- \\
0.770) \\
\end{array}$ \\
\hline \multicolumn{5}{|l|}{ Admissions via ED } \\
\hline Outcome & & qSOFA & NEWS & qNEWS \\
\hline \multirow{5}{*}{ In-hospital Mortality } & All admissions & \begin{tabular}{|l|}
0.691 \\
$(0.686-$ \\
$0.697)$ \\
\end{tabular} & $\begin{array}{l}0.830 \\
(0.825- \\
0.834)\end{array}$ & $\begin{array}{l}0.713 \\
(0.707- \\
0.719) \\
\end{array}$ \\
\hline & $\begin{array}{l}1^{\text {ry }} \text { infection } \\
\text { code }\end{array}$ & \begin{tabular}{|l|}
0.684 \\
$(0.675-$ \\
$0.692)$ \\
\end{tabular} & $\begin{array}{l}0.800 \\
(0.793- \\
0.807)\end{array}$ & $\begin{array}{l}0.702 \\
(0.692- \\
0.711)\end{array}$ \\
\hline & $\begin{array}{l}2^{\text {ry }} \text { infection } \\
\text { code }\end{array}$ & \begin{tabular}{|l|}
0.653 \\
$(0.640-$ \\
$0.666)$ \\
\end{tabular} & $\begin{array}{l}0.766 \\
(0.754- \\
0.778)\end{array}$ & $\begin{array}{l}0.675 \\
(0.661- \\
0.689)\end{array}$ \\
\hline & $\begin{array}{l}1^{\text {ry }} \text { or } 2^{\text {ry }} \\
\text { infection code }\end{array}$ & \begin{tabular}{|l|}
0.675 \\
$(0.668-$ \\
$0.682)$ \\
\end{tabular} & $\begin{array}{l}0.790 \\
(0.784- \\
0.796)\end{array}$ & $\begin{array}{l}0.694 \\
(0.686- \\
0.701)\end{array}$ \\
\hline & $\begin{array}{l}\text { No infection } \\
\text { code }\end{array}$ & \begin{tabular}{|l|}
0.705 \\
$(0.696-$ \\
$0.714)$ \\
\end{tabular} & $\begin{array}{l}0.844 \\
(0.836- \\
0.851) \\
\end{array}$ & $\begin{array}{l}0.731 \\
(0.721- \\
0.740) \\
\end{array}$ \\
\hline \multirow{3}{*}{$\begin{array}{r}\text { In-hospital Mortality or } \mathrm{ICU} \geq \\
3 \text { days }\end{array}$} & All admissions & \begin{tabular}{|l|}
0.691 \\
$(0.686-$ \\
$0.696)$ \\
\end{tabular} & $\begin{array}{l}0.831 \\
(0.827- \\
0.836)\end{array}$ & $\begin{array}{l}0.713 \\
(0.707- \\
0.719) \\
\end{array}$ \\
\hline & $\begin{array}{l}1^{\text {ry }} \text { infection } \\
\text { code }\end{array}$ & \begin{tabular}{|l|}
0.688 \\
$(0.680-$ \\
$0.696)$ \\
\end{tabular} & $\begin{array}{l}0.806 \\
(0.800- \\
0.813)\end{array}$ & $\begin{array}{l}0.707 \\
(0.698- \\
0.716) \\
\end{array}$ \\
\hline & $\begin{array}{l}2^{\text {ry }} \text { infection } \\
\text { code }\end{array}$ & \begin{tabular}{|l|}
0.652 \\
$(0.640-$ \\
$0.664)$
\end{tabular} & $\begin{array}{l}0.769 \\
(0.758- \\
0.780)\end{array}$ & $\begin{array}{l}0.675 \\
(0.662- \\
0.688)\end{array}$ \\
\hline
\end{tabular}




\begin{tabular}{|c|c|c|c|c|}
\hline & $\begin{array}{l}1^{\text {ry }} \text { or } 2^{\text {ry }} \\
\text { infection code }\end{array}$ & $\begin{array}{l}0.677 \\
(0.671- \\
0.684)\end{array}$ & $\begin{array}{l}0.794 \\
(0.789- \\
0.800)\end{array}$ & $\begin{array}{l}0.697 \\
(0.690- \\
0.704)\end{array}$ \\
\hline & $\begin{array}{l}\text { No infection } \\
\text { code }\end{array}$ & $\begin{array}{l}0.701 \\
(0.693- \\
0.710) \\
\end{array}$ & $\begin{array}{l}0.841 \\
(0.834- \\
0.848)\end{array}$ & $\begin{array}{l}0.726 \\
(0.717- \\
0.736) \\
\end{array}$ \\
\hline \multirow{5}{*}{ Mortality within 24 hours } & All admissions & $\begin{array}{l}0.803 \\
(0.795- \\
0.811)\end{array}$ & $\begin{array}{l}0.917 \\
(0.912- \\
0.923)\end{array}$ & $\begin{array}{l}0.829 \\
(0.820- \\
0.837)\end{array}$ \\
\hline & $\begin{array}{l}1^{\text {ry }} \text { infection } \\
\text { code }\end{array}$ & $\begin{array}{l}0.813 \\
(0.800- \\
0.826)\end{array}$ & $\begin{array}{l}0.913 \\
(0.904- \\
0.921)\end{array}$ & $\begin{array}{l}0.837 \\
(0.823- \\
0.850)\end{array}$ \\
\hline & $\begin{array}{l}2^{\text {ry }} \text { infection } \\
\text { code }\end{array}$ & $\begin{array}{l}0.792 \\
(0.768- \\
0.816)\end{array}$ & $\begin{array}{l}0.905 \\
(0.889- \\
0.921)\end{array}$ & $\begin{array}{l}0.820 \\
(0.795- \\
0.844)\end{array}$ \\
\hline & $\begin{array}{l}1^{\text {ry }} \text { or } 2^{\text {ry }} \\
\text { infection code }\end{array}$ & $\begin{array}{l}0.808 \\
(0.797- \\
0.820)\end{array}$ & $\begin{array}{l}0.911 \\
(0.904- \\
0.919)\end{array}$ & $\begin{array}{l}0.833 \\
(0.820- \\
0.845)\end{array}$ \\
\hline & $\begin{array}{l}\text { No infection } \\
\text { code }\end{array}$ & $\begin{array}{l}0.787 \\
(0.775- \\
0.799) \\
\end{array}$ & $\begin{array}{l}0.908 \\
(0.899- \\
0.917)\end{array}$ & $\begin{array}{l}0.814 \\
(0.801- \\
0.826) \\
\end{array}$ \\
\hline \multirow{5}{*}{$\begin{array}{r}\text { In-hospital Mortality or } \\
\text { unanticipated ICU admission }\end{array}$} & All admissions & $\begin{array}{l}0.685 \\
(0.681- \\
0.690)\end{array}$ & $\begin{array}{l}0.824 \\
(0.820- \\
0.828)\end{array}$ & $\begin{array}{l}0.707 \\
(0.702- \\
0.713)\end{array}$ \\
\hline & $\begin{array}{l}1^{\text {ry }} \text { infection } \\
\text { code }\end{array}$ & $\begin{array}{l}0.688 \\
(0.680- \\
0.696)\end{array}$ & $\begin{array}{l}0.806 \\
(0.799- \\
0.812)\end{array}$ & $\begin{array}{l}0.707 \\
(0.699- \\
0.716)\end{array}$ \\
\hline & $\begin{array}{l}2^{\text {ry }} \text { infection } \\
\text { code }\end{array}$ & $\begin{array}{l}0.645 \\
(0.634- \\
0.656) \\
\end{array}$ & $\begin{array}{l}0.762 \\
(0.751- \\
0.772) \\
\end{array}$ & $\begin{array}{l}0.666 \\
(0.654- \\
0.678) \\
\end{array}$ \\
\hline & $\begin{array}{l}1^{\text {ry }} \text { or } 2^{\text {ry }} \\
\text { infection code }\end{array}$ & $\begin{array}{l}0.675 \\
(0.668- \\
0.681)\end{array}$ & $\begin{array}{l}0.791 \\
(0.785- \\
0.796)\end{array}$ & $\begin{array}{l}0.694 \\
(0.687- \\
0.701)\end{array}$ \\
\hline & $\begin{array}{l}\text { No infection } \\
\text { code }\end{array}$ & $\begin{array}{l}0.690 \\
(0.682- \\
0.698) \\
\end{array}$ & $\begin{array}{l}0.827 \\
(0.820- \\
0.834) \\
\end{array}$ & $\begin{array}{l}0.714 \\
(0.705- \\
0.722) \\
\end{array}$ \\
\hline \multirow{5}{*}{$\begin{array}{r}\text { Mortality within } 24 \text { hours or } \\
\text { unanticipated ICU admission } \\
\text { within } 24 \text { hours }\end{array}$} & All admissions & $\begin{array}{l}0.775 \\
(0.768- \\
0.782)\end{array}$ & $\begin{array}{l}0.895 \\
(0.890- \\
0.900)\end{array}$ & $\begin{array}{l}0.801 \\
(0.793- \\
0.808)\end{array}$ \\
\hline & $\begin{array}{l}1^{\text {ry }} \text { infection } \\
\text { code }\end{array}$ & $\begin{array}{l}0.796 \\
(0.785- \\
0.808)\end{array}$ & $\begin{array}{l}0.900 \\
(0.893- \\
0.908)\end{array}$ & $\begin{array}{l}0.823 \\
(0.811- \\
0.834)\end{array}$ \\
\hline & $\begin{array}{l}2^{r y} \text { infection } \\
\text { code }\end{array}$ & $\begin{array}{l}0.744 \\
(0.726- \\
0.763) \\
\end{array}$ & $\begin{array}{l}0.865 \\
(0.850- \\
0.879)\end{array}$ & $\begin{array}{l}0.770 \\
(0.751- \\
0.790)\end{array}$ \\
\hline & $\begin{array}{l}1^{\text {ry }} \text { or } 2^{\text {ry }} \\
\text { infection code }\end{array}$ & $\begin{array}{l}0.781 \\
(0.772- \\
0.791) \\
\end{array}$ & $\begin{array}{l}0.889 \\
(0.882- \\
0.896)\end{array}$ & $\begin{array}{l}0.807 \\
(0.797- \\
0.818) \\
\end{array}$ \\
\hline & $\begin{array}{l}\text { No infection } \\
\text { code }\end{array}$ & $\begin{array}{l}0.753 \\
(0.742- \\
0.763) \\
\end{array}$ & $\begin{array}{l}0.879 \\
(0.871- \\
0.888)\end{array}$ & $\begin{array}{l}0.779 \\
(0.767- \\
0.790)\end{array}$ \\
\hline \multicolumn{5}{|l|}{ Direct Admissions } \\
\hline OUTCOME & & qSOFA & NEWS & qNEWS \\
\hline
\end{tabular}




\begin{tabular}{|c|c|c|c|c|}
\hline \multirow{5}{*}{ In-hospital Mortality } & All admissions & $\begin{array}{l}0.653 \\
(0.644- \\
0.662)\end{array}$ & $\begin{array}{l}0.805 \\
(0.796- \\
0.813)\end{array}$ & $\begin{array}{l}0.672 \\
(0.662- \\
0.682)\end{array}$ \\
\hline & $\begin{array}{l}1^{\text {ry }} \text { infection } \\
\text { code }\end{array}$ & $\begin{array}{l}0.654 \\
(0.639- \\
0.670)\end{array}$ & $\begin{array}{l}0.800 \\
(0.786- \\
0.813)\end{array}$ & $\begin{array}{l}0.671 \\
(0.653- \\
0.687) \\
\end{array}$ \\
\hline & $\begin{array}{l}2^{\text {ry }} \text { infection } \\
\text { code }\end{array}$ & $\begin{array}{l}0.622 \\
(0.603- \\
0.640)\end{array}$ & $\begin{array}{l}0.741 \\
(0.722- \\
0.760) \\
\end{array}$ & $\begin{array}{l}0.638 \\
(0.617- \\
0.659) \\
\end{array}$ \\
\hline & $\begin{array}{l}1^{\text {ry }} \text { or } 2^{\text {ry }} \\
\text { infection code }\end{array}$ & $\begin{array}{l}0.642 \\
(0.630- \\
0.653)\end{array}$ & $\begin{array}{l}0.778 \\
(0.767- \\
0.789)\end{array}$ & $\begin{array}{l}0.657 \\
(0.644- \\
0.670)\end{array}$ \\
\hline & $\begin{array}{l}\text { No infection } \\
\text { code }\end{array}$ & $\begin{array}{l}0.654 \\
(0.641- \\
0.667) \\
\end{array}$ & $\begin{array}{l}0.806 \\
(0.793- \\
0.818) \\
\end{array}$ & $\begin{array}{l}0.674 \\
(0.659- \\
0.689) \\
\end{array}$ \\
\hline \multirow{5}{*}{$\begin{array}{r}\text { In-hospital Mortality or ICU } \geq \\
3 \text { days }\end{array}$} & All admissions & $\begin{array}{l}0.647 \\
(0.639- \\
0.655)\end{array}$ & $\begin{array}{l}0.799 \\
(0.792- \\
0.807)\end{array}$ & $\begin{array}{l}0.665 \\
(0.656- \\
0.674)\end{array}$ \\
\hline & $\begin{array}{l}1^{\text {ry }} \text { infection } \\
\text { code }\end{array}$ & $\begin{array}{l}0.654 \\
(0.641- \\
0.668)\end{array}$ & $\begin{array}{l}0.802 \\
(0.789- \\
0.814)\end{array}$ & $\begin{array}{l}0.670 \\
(0.655- \\
0.686) \\
\end{array}$ \\
\hline & $\begin{array}{l}2^{\text {ry }} \text { infection } \\
\text { code }\end{array}$ & $\begin{array}{l}0.610 \\
(0.595- \\
0.626)\end{array}$ & $\begin{array}{l}0.738 \\
(0.722- \\
0.754)\end{array}$ & $\begin{array}{l}0.625 \\
(0.607- \\
0.642)\end{array}$ \\
\hline & $\begin{array}{l}1^{\text {ry }} \text { or } 2^{\text {ry }} \\
\text { infection code }\end{array}$ & $\begin{array}{l}0.635 \\
(0.625- \\
0.646)\end{array}$ & $\begin{array}{l}0.776 \\
(0.766- \\
0.786)\end{array}$ & $\begin{array}{l}0.650 \\
(0.638- \\
0.661)\end{array}$ \\
\hline & $\begin{array}{l}\text { No infection } \\
\text { code }\end{array}$ & $\begin{array}{l}0.648 \\
(0.636- \\
0.661)\end{array}$ & $\begin{array}{l}0.797 \\
(0.785- \\
0.809)\end{array}$ & $\begin{array}{l}0.668 \\
(0.654- \\
0.682)\end{array}$ \\
\hline \multirow{5}{*}{ Mortality within 24 hours } & All admissions & $\begin{array}{l}0.774 \\
(0.756- \\
0.791)\end{array}$ & $\begin{array}{c}0.907 \\
(0.895- \\
0.918)\end{array}$ & $\begin{array}{c}0.800 \\
(0.782- \\
0.819)\end{array}$ \\
\hline & $\begin{array}{l}1^{\text {ry }} \text { infection } \\
\text { code }\end{array}$ & $\begin{array}{l}0.793 \\
(0.763- \\
0.824)\end{array}$ & $\begin{array}{c}0.920 \\
(0.901- \\
0.938)\end{array}$ & $\begin{array}{c}0.818 \\
(0.785- \\
0.851)\end{array}$ \\
\hline & $\begin{array}{l}2^{\text {ry }} \text { infection } \\
\text { code }\end{array}$ & $\begin{array}{l}0.780 \\
(0.731- \\
0.829)\end{array}$ & $\begin{array}{c}0.904 \\
(0.870- \\
0.934)\end{array}$ & $\begin{array}{c}0.801 \\
(0.749- \\
0.853)\end{array}$ \\
\hline & $\begin{array}{l}1^{\text {ry }} \text { or } 2^{\text {ry }} \\
\text { infection code }\end{array}$ & $\begin{array}{l}0.789 \\
(0.763- \\
0.815)\end{array}$ & $\begin{array}{c}0.915 \\
(0.898- \\
0.930) \\
\end{array}$ & $\begin{array}{c}0.813 \\
(0.785- \\
0.840) \\
\end{array}$ \\
\hline & $\begin{array}{l}\text { No infection } \\
\text { code }\end{array}$ & $\begin{array}{l}0.753 \\
(0.730- \\
0.776)\end{array}$ & $\begin{array}{c}0.890 \\
(0.873- \\
0.907)\end{array}$ & $\begin{array}{c}0.782 \\
(0.757- \\
0.806)\end{array}$ \\
\hline \multirow{4}{*}{$\begin{array}{l}\text { In-hospital Mortality or } \\
\text { unanticipated ICU admission }\end{array}$} & All admissions & $\begin{array}{l}0.638 \\
(0.631- \\
0.645)\end{array}$ & $\begin{array}{c}0.784 \\
(0.777- \\
0.791)\end{array}$ & $\begin{array}{c}0.652 \\
(0.644- \\
0.660)\end{array}$ \\
\hline & $\begin{array}{l}1^{\text {ry }} \text { infection } \\
\text { code }\end{array}$ & $\begin{array}{l}0.654 \\
(0.641- \\
0.666)\end{array}$ & $\begin{array}{c}0.799 \\
(0.788- \\
0.810)\end{array}$ & $\begin{array}{c}0.669 \\
(0.654- \\
0.683)\end{array}$ \\
\hline & $\begin{array}{l}2^{\text {ry }} \text { infection } \\
\text { code }\end{array}$ & $\begin{array}{l}0.603 \\
(0.589- \\
0.617)\end{array}$ & $\begin{array}{c}0.728 \\
(0.714- \\
0.743) \\
\end{array}$ & $\begin{array}{c}0.614 \\
(0.598- \\
0.629) \\
\end{array}$ \\
\hline & $\begin{array}{l}1^{\text {ry }} \text { or } 2^{\text {ry }} \\
\text { infection code }\end{array}$ & $\begin{array}{l}0.631 \\
(0.622- \\
0.641)\end{array}$ & $\begin{array}{c}0.770 \\
(0.761- \\
0.779)\end{array}$ & $\begin{array}{c}0.643 \\
(0.632- \\
0.654)\end{array}$ \\
\hline
\end{tabular}




\begin{tabular}{|c|c|c|c|c|}
\hline & $\begin{array}{l}\text { No infection } \\
\text { code }\end{array}$ & $\begin{array}{l}0.632 \\
(0.621- \\
0.643)\end{array}$ & $\begin{array}{l}0.768 \\
(0.757- \\
0.779) \\
\end{array}$ & $\begin{array}{c}0.647 \\
(0.635- \\
0.659) \\
\end{array}$ \\
\hline \multirow{5}{*}{$\begin{array}{r}\text { Mortality within } 24 \text { hours or } \\
\text { unanticipated ICU admission } \\
\text { within } 24 \text { hours }\end{array}$} & All admissions & $\begin{array}{l}0.720 \\
(0.708- \\
0.732)\end{array}$ & $\begin{array}{c}0.851 \\
(0.841- \\
0.861) \\
\end{array}$ & $\begin{array}{l}0.740 \\
(0.726- \\
0.754) \\
\end{array}$ \\
\hline & $\begin{array}{l}1^{r y} \text { infection } \\
\text { code }\end{array}$ & $\begin{array}{l}0.750 \\
(0.728- \\
0.772)\end{array}$ & $\begin{array}{c}0.886 \\
(0.871- \\
0.901)\end{array}$ & $\begin{array}{c}0.774 \\
(0.750- \\
0.798)\end{array}$ \\
\hline & $\begin{array}{l}2^{\text {ry }} \text { infection } \\
\text { code }\end{array}$ & $\begin{array}{l}0.692 \\
(0.664- \\
0.721)\end{array}$ & $\begin{array}{l}0.819 \\
(0.796- \\
0.842)\end{array}$ & $\begin{array}{l}0.706 \\
(0.674- \\
0.737)\end{array}$ \\
\hline & $\begin{array}{l}1^{\text {ry }} \text { or } 2^{\text {ry }} \\
\text { infection code }\end{array}$ & $\begin{array}{l}0.727 \\
(0.710- \\
0.745) \\
\end{array}$ & $\begin{array}{c}0.861 \\
(0.848- \\
0.874) \\
\end{array}$ & $\begin{array}{l}0.747 \\
(0.727- \\
0.765) \\
\end{array}$ \\
\hline & $\begin{array}{l}\text { No infection } \\
\text { code }\end{array}$ & $\begin{array}{l}0.700 \\
(0.682- \\
0.717)\end{array}$ & $\begin{array}{l}0.816 \\
(0.800- \\
0.832)\end{array}$ & $\begin{array}{l}0.719 \\
(0.700- \\
0.738)\end{array}$ \\
\hline
\end{tabular}


Supplementary Table 5: Area under the receiver operator characteristics curve for quick Sequential [Sepsis-related] Organ Failure Assessment (qSOFA) score, the National Early Warning Score (NEWS) and the quick National Early Warning Score (qNEWS) for a range of outcomes for all admissions, including those where there were no vital signs observations taken in the 24 hours prior to discharge.

\begin{tabular}{|c|c|c|c|}
\hline All admissions & & qSOFA & 1 \\
\hline Outcome & & & \\
\hline \multirow{5}{*}{ In-hospital Mortality } & All admissions & $0.670(0.666-0.674)$ & \\
\hline & $1^{\text {ry }}$ infection code & $0.666(0.660-0.672)$ & 0 \\
\hline & $2^{\text {ry }}$ infection code & $0.636(0.628-0.644)$ & \\
\hline & $1^{\text {ry }}$ or $2^{\text {ry }}$ infection code & $0.655(0.651-0.660)$ & \\
\hline & No infection code & $0.677(0.671-0.682)$ & \\
\hline \multirow{5}{*}{ In-hospital Mortality or ICU $\geq 3$ days } & All admissions & $0.669(0.666-0.673)$ & 0 \\
\hline & $1^{\text {ry }}$ infection code & $0.669(0.664-0.675)$ & 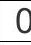 \\
\hline & $2^{\text {ry }}$ infection code & 0.634 (0.627-0.642) & 0 \\
\hline & $1^{\text {ry }}$ or $2^{\text {ry }}$ infection code & $0.656(0.652-0.661)$ & 0 \\
\hline & No infection code & $0.674(0.668-0.680)$ & \\
\hline \multirow{5}{*}{ Mortality within 24 hours } & All admissions & $0.795(0.788-0.802)$ & 0 \\
\hline & $1^{\text {ry }}$ infection code & $0.806(0.793-0.818)$ & 0 \\
\hline & $2^{\text {ry }}$ infection code & $0.783(0.761-0.804)$ & 0 \\
\hline & $1^{\text {ry }}$ or $2^{\text {ry }}$ infection code & $0.800(0.789-0.810)$ & - \\
\hline & No infection code & $0.777(0.766-0.788)$ & \\
\hline \multirow{5}{*}{ In-hospital Mortality or unanticipated ICU admission } & All admissions & $0.665(0.662-0.668)$ & 0 \\
\hline & $1^{\text {ry }}$ infection code & $0.670(0.664-0.675)$ & 0 \\
\hline & $2^{\text {ry }}$ infection code & $0.630(0.622-0.637)$ & 0 \\
\hline & $1^{\text {ry }}$ or $2^{\text {ry }}$ infection code & $0.655(0.650-0.659)$ & 0 \\
\hline & No infection code & $0.666(0.661-0.671)$ & \\
\hline \multirow{5}{*}{$\begin{array}{r}\text { In-hospital Mortality or unanticipated ICU admission within } \\
24 \text { hours }\end{array}$} & All admissions & $0.756(0.750-0.762)$ & 0 \\
\hline & $1^{\text {ry }}$ infection code & $0.781(0.772-0.790)$ & 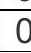 \\
\hline & $2^{\text {ry }}$ infection code & $0.722(0.708-0.737)$ & 0 \\
\hline & $1^{\text {ry }}$ or $2^{\text {ry }}$ infection code & $0.762(0.754-0.769)$ & \\
\hline & No infection code & $0.732(0.723-0.741)$ & 0 \\
\hline
\end{tabular}

Supplementary Table 6. Sensitivity, false positive rates and positive predictive values at each threshold of NEWS/qSOFA for the primary outcome of in-hospital mortality

\begin{tabular}{|c|c|c|c|c|c|c|c|}
\hline & & \multicolumn{3}{|c|}{ NEWS } & \multicolumn{3}{|c|}{ qSOFA } \\
\hline Group & Threshold & $\begin{array}{c}\text { Sensitivity } \\
(\%)\end{array}$ & $\begin{array}{l}\text { False } \\
\text { positive } \\
\text { rate (\%) }\end{array}$ & $\begin{array}{c}\text { Positive } \\
\text { Predictive } \\
\text { Value (\%) }\end{array}$ & $\begin{array}{c}\text { Sensitivity } \\
(\%)\end{array}$ & $\begin{array}{l}\text { False } \\
\text { positive } \\
\text { rate (\%) }\end{array}$ & $\begin{array}{c}\text { Positive } \\
\text { Predictive } \\
\text { Value (\%) }\end{array}$ \\
\hline All admissions & 0 & 100 & 100 & 0.638 & 100 & 100 & 1.72 \\
\hline All admissions & 1 & 91.5 & 61.6 & 1.04 & 46.6 & 11.7 & 7.97 \\
\hline All admissions & 2 & 81.4 & 34 & 2 & 12.5 & 0.326 & 48.7 \\
\hline All admissions & 3 & 69.2 & 16.8 & 3.84 & 2.08 & 0.00787 & 88.5 \\
\hline All admissions & 4 & 57.2 & 8.08 & 7.1 & & & \\
\hline All admissions & 5 & 46.6 & 4.07 & 12.5 & & & \\
\hline All admissions & 6 & 36.2 & 1.97 & 19.8 & & & \\
\hline All admissions & 7 & 27.1 & 0.916 & 29.3 & & & \\
\hline All admissions & 8 & 20.5 & 0.456 & 41.4 & & & \\
\hline All admissions & 9 & 14.5 & 0.21 & 52 & & & \\
\hline All admissions & 10 & 10.1 & 0.0936 & 64.5 & & & \\
\hline All admissions & 11 & 6.78 & 0.0399 & 74.8 & & & \\
\hline All admissions & 12 & 4.4 & 0.0167 & 83.6 & & & \\
\hline
\end{tabular}




\begin{tabular}{|c|c|c|c|c|c|c|c|}
\hline All admissions & 13 & 2.76 & 0.00737 & 88.4 & & & \\
\hline All admissions & 14 & 1.57 & 0.00288 & 94.1 & & & \\
\hline $\begin{array}{r}\text { Admissions } \\
\text { with a 1ry } \\
\text { infection code }\end{array}$ & 0 & 100 & 100 & 1.66 & 100 & 100 & 4.21 \\
\hline $\begin{array}{r}\text { Admissions } \\
\text { with a 1ry } \\
\text { infection code }\end{array}$ & 1 & 92.9 & 69.4 & 2.45 & 48.7 & 14.9 & 15.3 \\
\hline $\begin{array}{r}\text { Admissions } \\
\text { with a 1ry } \\
\text { infection code }\end{array}$ & 2 & 84.2 & 44 & 4.11 & 13.5 & 0.655 & 55.5 \\
\hline $\begin{array}{r}\text { Admissions } \\
\text { with a 1ry } \\
\text { infection code }\end{array}$ & 3 & 73.3 & 25.5 & 7.08 & 2.77 & 0.0261 & 88.6 \\
\hline $\begin{array}{r}\text { Admissions } \\
\text { with a 1ry } \\
\text { infection code }\end{array}$ & 4 & 61.8 & 14.5 & 11.1 & & & \\
\hline $\begin{array}{r}\text { Admissions } \\
\text { with a 1ry } \\
\text { infection code }\end{array}$ & 5 & 51.4 & 8.38 & 16.8 & & & \\
\hline $\begin{array}{r}\text { Admissions } \\
\text { with a 1ry } \\
\text { infection code }\end{array}$ & 6 & 40.6 & 4.46 & 24.1 & & & \\
\hline $\begin{array}{r}\text { Admissions } \\
\text { with a 1ry } \\
\text { infection code }\end{array}$ & 7 & 31 & 2.25 & 33.3 & & & \\
\hline $\begin{array}{r}\text { Admissions } \\
\text { with a 1ry } \\
\text { infection code }\end{array}$ & 8 & 23.8 & 1.21 & 45.4 & & & \\
\hline $\begin{array}{r}\text { Admissions } \\
\text { with a 1ry } \\
\text { infection code }\end{array}$ & 9 & 16.9 & 0.6 & 52 & & & \\
\hline $\begin{array}{r}\text { Admissions } \\
\text { with a 1ry } \\
\text { infection code }\end{array}$ & 10 & 12.1 & 0.278 & 64 & & & \\
\hline $\begin{array}{r}\text { Admissions } \\
\text { with a } 1 r y \\
\text { infection code }\end{array}$ & 11 & 8.36 & 0.123 & 75.9 & & & \\
\hline $\begin{array}{r}\text { Admissions } \\
\text { with a } 1 r y \\
\text { infection code }\end{array}$ & 12 & 5.42 & 0.0552 & 82.6 & & & \\
\hline $\begin{array}{r}\text { Admissions } \\
\text { with a } 1 r y \\
\text { infection code }\end{array}$ & 13 & 3.39 & 0.0241 & 87.5 & & & \\
\hline $\begin{array}{r}\text { Admissions } \\
\text { with a 1ry } \\
\text { infection code }\end{array}$ & 14 & 2.01 & 0.0096 & 93.9 & & & \\
\hline $\begin{array}{r}\text { Admissions } \\
\text { with a } 2 r y \\
\text { infection code }\end{array}$ & 0 & 100 & 100 & 2.19 & 100 & 100 & 4.53 \\
\hline $\begin{array}{r}\text { Admissions } \\
\text { with a } 2 r y \\
\text { infection code }\end{array}$ & 1 & 89.3 & 66.6 & 3.15 & 41.2 & 13.8 & 14.1 \\
\hline $\begin{array}{r}\text { Admissions } \\
\text { with a } 2 r y \\
\text { infection code }\end{array}$ & 2 & 77.3 & 41 & 5.23 & 10.1 & 0.639 & 50 \\
\hline $\begin{array}{r}\text { Admissions } \\
\text { with a } 2 r y \\
\text { infection code }\end{array}$ & 3 & 63.2 & 23.3 & 7.76 & 1.18 & 0.0134 & 86.1 \\
\hline $\begin{array}{r}\text { Admissions } \\
\text { with a } 2 r y \\
\text { infection code }\end{array}$ & 4 & 50.3 & 12.6 & 11.7 & & & \\
\hline $\begin{array}{r}\text { Admissions } \\
\text { with a } 2 r y \\
\text { infection code }\end{array}$ & 5 & 39.5 & 6.93 & 17.2 & & & \\
\hline $\begin{array}{r}\text { Admissions } \\
\text { with a } 2 r y \\
\text { infection code }\end{array}$ & 6 & 29.5 & 3.59 & 22.7 & & & \\
\hline $\begin{array}{r}\text { Admissions } \\
\text { with a } 2 r y \\
\text { infection code }\end{array}$ & 7 & 21.7 & 1.74 & 31.8 & & & \\
\hline
\end{tabular}




\begin{tabular}{|c|c|c|c|c|c|c|c|}
\hline $\begin{array}{r}\text { Admissions } \\
\text { with a } 2 r y \\
\text { infection code }\end{array}$ & 8 & 16.1 & 0.913 & 41.2 & & & \\
\hline $\begin{array}{r}\text { Admissions } \\
\text { with a } 2 r y \\
\text { infection code }\end{array}$ & 9 & 11.4 & 0.439 & 52.3 & & & \\
\hline $\begin{array}{r}\text { Admissions } \\
\text { with a } 2 r y \\
\text { infection code }\end{array}$ & 10 & 7.74 & 0.209 & 62.5 & & & \\
\hline $\begin{array}{r}\text { Admissions } \\
\text { with a } 2 r y \\
\text { infection code }\end{array}$ & 11 & 4.85 & 0.0881 & 72.2 & & & \\
\hline $\begin{array}{r}\text { Admissions } \\
\text { with a } 2 r y \\
\text { infection code }\end{array}$ & 12 & 2.88 & 0.035 & 83.1 & & & \\
\hline $\begin{array}{r}\text { Admissions } \\
\text { with a } 2 r y \\
\text { infection code }\end{array}$ & 13 & 1.75 & 0.0189 & 81.2 & & & \\
\hline $\begin{array}{r}\text { Admissions } \\
\text { with a } 2 r y \\
\text { infection code }\end{array}$ & 14 & 1.07 & 0.00795 & 90.5 & & & \\
\hline $\begin{array}{r}\text { Admissions } \\
\text { with either 1ry } \\
\text { or } 2 r y \text { infection } \\
\text { code }\end{array}$ & 0 & 100 & 100 & 1.84 & 100 & 100 & 4.32 \\
\hline $\begin{array}{r}\text { Admissions } \\
\text { with either 1ry } \\
\text { or } 2 r y \text { infection } \\
\text { code }\end{array}$ & 1 & 91.8 & 68.5 & 2.68 & 46.3 & 14.5 & 14.9 \\
\hline $\begin{array}{r}\text { Admissions } \\
\text { with either 1ry } \\
\text { or } 2 r y \text { infection } \\
\text { code }\end{array}$ & 2 & 82 & 43 & 4.47 & 12.5 & 0.65 & 53.8 \\
\hline $\begin{array}{r}\text { Admissions } \\
\text { with either 1ry } \\
\text { or } 2 r y \text { infection } \\
\text { code }\end{array}$ & 3 & 70.1 & 24.7 & 7.3 & 2.27 & 0.022 & 88.1 \\
\hline $\begin{array}{r}\text { Admissions } \\
\text { with either 1ry } \\
\text { or } 2 r y \text { infection } \\
\text { code }\end{array}$ & 4 & 58.2 & 13.9 & 11.3 & & & \\
\hline $\begin{array}{r}\text { Admissions } \\
\text { with either 1ry } \\
\text { or } 2 r y \text { infection } \\
\text { code }\end{array}$ & 5 & 47.6 & 7.91 & 16.9 & & & \\
\hline $\begin{array}{r}\text { Admissions } \\
\text { with either 1ry } \\
\text { or } 2 r y \text { infection } \\
\text { code }\end{array}$ & 6 & 37.1 & 4.18 & 23.7 & & & \\
\hline $\begin{array}{r}\text { Admissions } \\
\text { with either 1ry } \\
\text { or } 2 r y \text { infection } \\
\text { code }\end{array}$ & 7 & 28 & 2.09 & 32.9 & & & \\
\hline $\begin{array}{r}\text { Admissions } \\
\text { with either 1ry } \\
\text { or } 2 r y \text { infection } \\
\text { code }\end{array}$ & 8 & 21.4 & 1.11 & 44.3 & & & \\
\hline $\begin{array}{r}\text { Admissions } \\
\text { with either 1ry } \\
\text { or } 2 r y \text { infection } \\
\text { code }\end{array}$ & 9 & 15.2 & 0.548 & 52 & & & \\
\hline $\begin{array}{r}\text { Admissions } \\
\text { with either 1ry } \\
\text { or } 2 r y \text { infection } \\
\text { code }\end{array}$ & 10 & 10.7 & 0.255 & 63.6 & & & \\
\hline $\begin{array}{r}\text { Admissions } \\
\text { with either 1ry } \\
\text { or } 2 r y \text { infection } \\
\text { code }\end{array}$ & 11 & 7.25 & 0.112 & 75 & & & \\
\hline $\begin{array}{r}\text { Admissions } \\
\text { with either 1ry } \\
\text { or } 2 r y \text { infection } \\
\text { code }\end{array}$ & 12 & 4.61 & 0.0486 & 82.7 & & & \\
\hline
\end{tabular}




\begin{tabular}{|c|c|c|c|c|c|c|c|}
\hline $\begin{array}{r}\text { Admissions } \\
\text { with either } 1 r y \\
\text { or } 2 r y \text { infection } \\
\text { code }\end{array}$ & 13 & 2.87 & 0.0224 & 86.3 & & & \\
\hline $\begin{array}{r}\text { Admissions } \\
\text { with either 1ry } \\
\text { or } 2 r y \text { infection } \\
\text { code } \\
\end{array}$ & 14 & 1.71 & 0.00906 & 93.2 & & & \\
\hline $\begin{array}{r}\text { Admissions } \\
\text { with no 1ry or } \\
2 r y \text { infection } \\
\text { code }\end{array}$ & 0 & 100 & 100 & 0.303 & 100 & 100 & 0.801 \\
\hline $\begin{array}{r}\text { Admissions } \\
\text { with no 1ry or } \\
2 r y \text { infection } \\
\text { code }\end{array}$ & 1 & 90.9 & 59.1 & 0.507 & 47 & 10.7 & 4.29 \\
\hline $\begin{array}{r}\text { Admissions } \\
\text { with no 1ry or } \\
2 r y \text { infection } \\
\text { code }\end{array}$ & 2 & 80.3 & 30.9 & 1.02 & 12.6 & 0.21 & 41.6 \\
\hline $\begin{array}{r}\text { Admissions } \\
\text { with no 1ry or } \\
2 r y \text { infection } \\
\text { code }\end{array}$ & 3 & 67.5 & 13.9 & 2.06 & 1.74 & 0.00285 & 89.4 \\
\hline $\begin{array}{r}\text { Admissions } \\
\text { with no 1ry or } \\
2 r y \text { infection } \\
\text { code }\end{array}$ & 4 & 55.3 & 6.01 & 4.21 & & & \\
\hline $\begin{array}{r}\text { Admissions } \\
\text { with no 1ry or } \\
2 r y \text { infection } \\
\text { code }\end{array}$ & 5 & 44.6 & 2.7 & 8.34 & & & \\
\hline $\begin{array}{r}\text { Admissions } \\
\text { with no } 1 r y \text { or } \\
2 r y \text { infection } \\
\text { code }\end{array}$ & 6 & 34.4 & 1.18 & 15.2 & & & \\
\hline $\begin{array}{r}\text { Admissions } \\
\text { with no } 1 r y \text { or } \\
2 r y \text { infection } \\
\text { code }\end{array}$ & 7 & 25.5 & 0.499 & 24.3 & & & \\
\hline $\begin{array}{r}\text { Admissions } \\
\text { with no } 1 r y \text { or } \\
2 r y \text { infection } \\
\text { code }\end{array}$ & 8 & 19 & 0.223 & 36.5 & & & \\
\hline $\begin{array}{r}\text { Admissions } \\
\text { with no 1ry or } \\
2 r y \text { infection } \\
\text { code }\end{array}$ & 9 & 13.4 & 0.0902 & 52.1 & & & \\
\hline $\begin{array}{r}\text { Admissions } \\
\text { with no 1ry or } \\
2 r y \text { infection } \\
\text { code }\end{array}$ & 10 & 9.03 & 0.036 & 66.3 & & & \\
\hline $\begin{array}{r}\text { Admissions } \\
\text { with no 1ry or } \\
2 r y \text { infection } \\
\text { code }\end{array}$ & 11 & 5.9 & 0.0143 & 74.4 & & & \\
\hline $\begin{array}{r}\text { Admissions } \\
\text { with no 1ry or } \\
2 r y \text { infection } \\
\text { code }\end{array}$ & 12 & 4.01 & 0.00538 & 85.7 & & & \\
\hline $\begin{array}{r}\text { Admissions } \\
\text { with no 1ry or } \\
2 r y \text { infection } \\
\text { code }\end{array}$ & 13 & 2.54 & 0.00202 & 92.7 & & & \\
\hline $\begin{array}{r}\text { Admissions } \\
\text { with no 1ry or } \\
2 r y \text { infection } \\
\text { code }\end{array}$ & 14 & 1.31 & 0.000681 & 96.4 & & & \\
\hline
\end{tabular}


